\title{
¿Continuidad o abandono? - El centro ceremonial de Tazumal, El Salvador C.A. después de la erupción del Volcán Ilopango entre el Siglo V - VI d.C.
}

DOI: http://dx.doi.org/10.5377/koot.v0i10.6702 URI: http://hdl.handle.net/11298/788

Lic. Shione Shibata y Rocío Herrera

Arqueólogos

shione911@yahoo.co.jp

\section{Resumen}

ruby5000_50@hotmail.com

La erupción del Volcán Ilopango, ocurrida entre los principios del siglo V y VI d.C., fue un evento catastrófico el cual provocó el abandono de la extensa área del Sureste de Mesoamérica según los estudios geológicos, sin embargo, conforme a los estudios vulcanológicos y los datos estratigráficos de las excavaciones arqueológicas en el sitio arqueológico Tazumal, cuya centro prehispánico no fue abandonado completamente por siglos, sino que, la población se habría recuperado en un corto plazo después de la erupción.

Palabras clave: Cultura; Tazumal; Geología; Arqueología; Vulcanismo; Ilopango - Volcanes.

\begin{abstract}
The eruption of the Ilopango Volcano, which occurred between the beginnings of the 5th and $6^{\text {th }}$ centuries $\mathrm{AD}$, was a catastrophic event which caused the abandonment of the extensive area of Southeastern Mesoamerica in accordance with geological studies, however, according to volcanological studies and the stratigraphic data of the archaeological excavations at the Tazumal archaeological site, this pre-Hispanic center had not been abandoned for centuries, yet, the population would have recovered in a short term after the eruption.
\end{abstract}

Keywords: Culture; Tazumal; Geology; Archeology; Vulcanism; Ilopango Volcanoes. 


\section{Introducción}

El actual Lago de Ilopango, en épocas pasadas fue la ubicación del Volcán Ilopango que está situado a $10 \mathrm{~km}$ al Este de la Capital San Salvador (Fig.1; Foto.1). Su caldera, se ha estimado con dimensiones aproximadas de $8.5 \mathrm{~km}$ de Norte a Sur y $11.3 \mathrm{~km}$ de Este a Oeste. Dicho Volcán tuvo diversas erupciones a lo largo de su historia, una de las cuales podría estimarse entre los Siglos V y VI d.C. considerándose muy catastrófica y afectando extensamente la vida humana, según diversos geólogos y arqueólogos (Sheets, 1983; Dull et.al., 2001; Dull, 2004; Dull et.al., 2010).

\section{Figura 1}

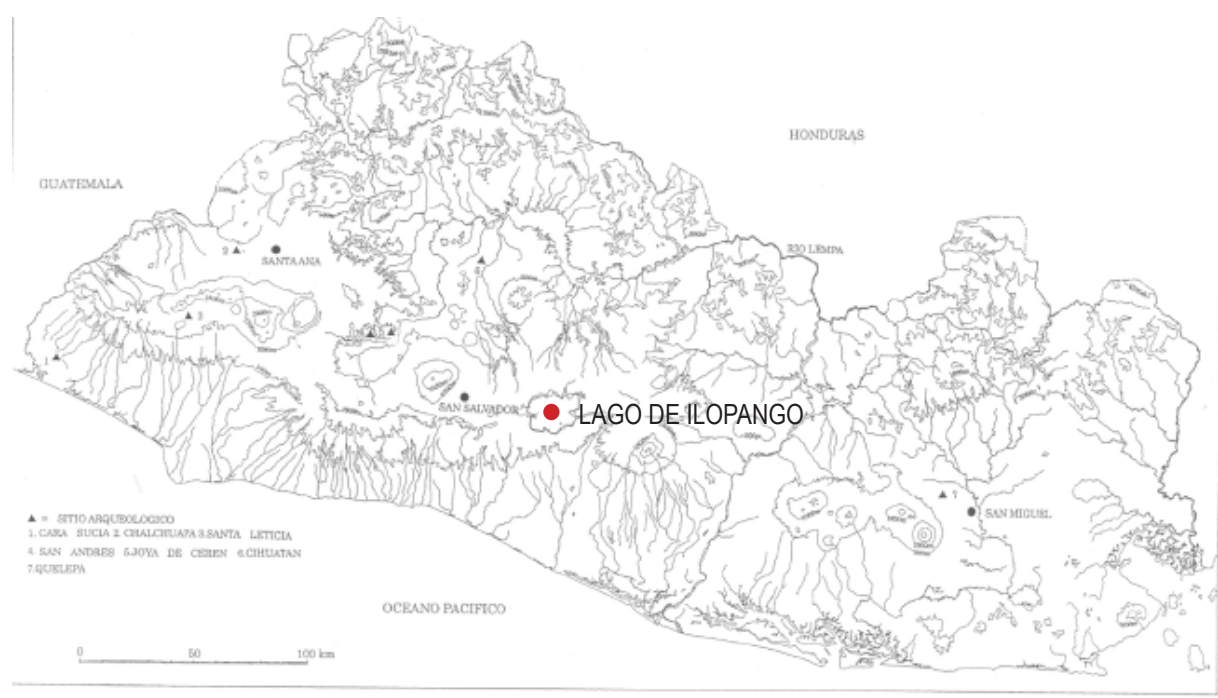

Ubicación del Lago de Ilopango en el territorio salvadoreño. 


\section{Fotografia 1}

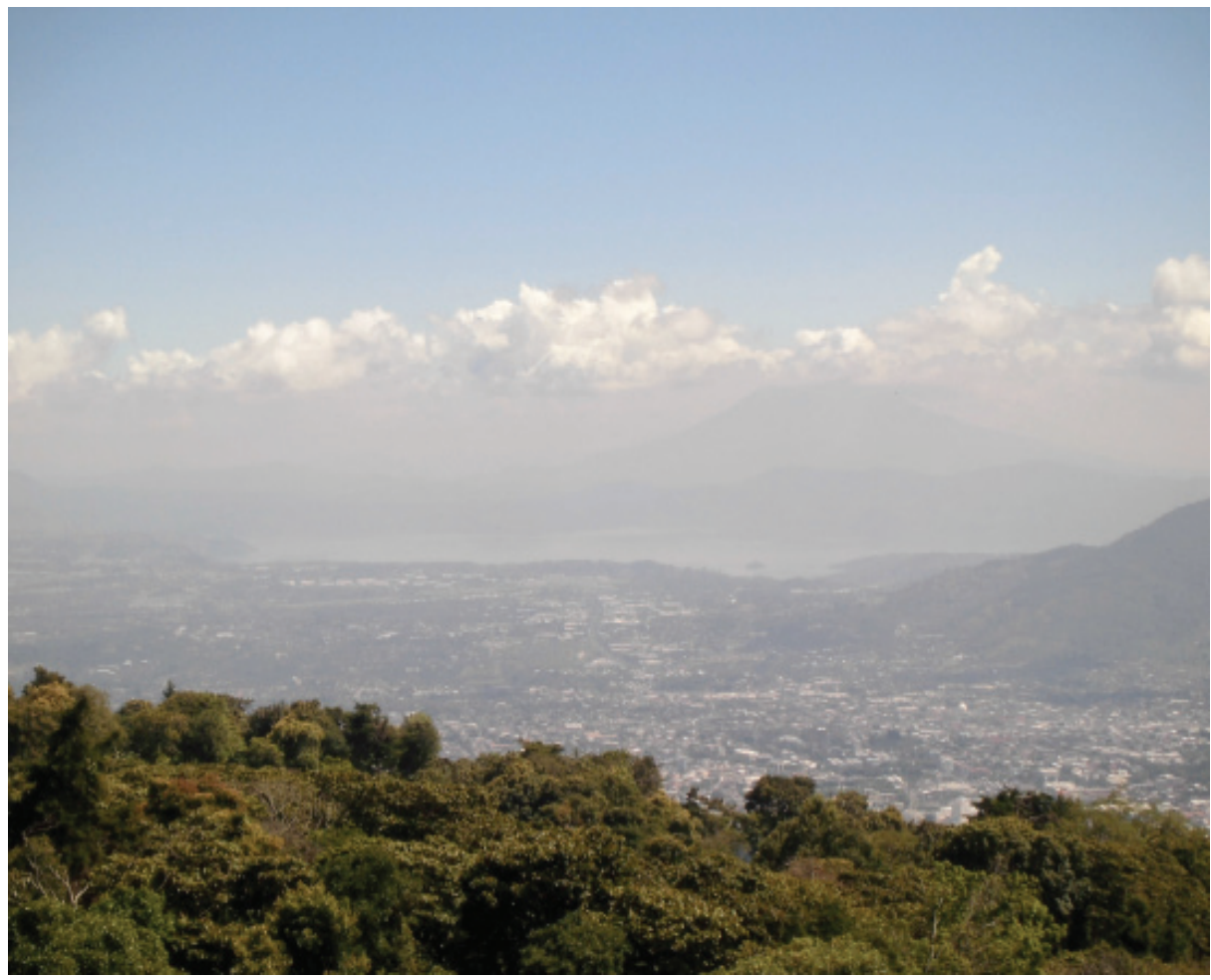

Lago de Ilopango (Vista hacia el Norte).

Alrededor del Lago de Ilopango se observan más de $20 \mathrm{~m}$ de grosor de la capa de la ceniza volcánica denominada como Tierra Blanca Joven (TBJ) por geólogos. Técnicos de la Administración Nacional de Acueductos y Alcantarillados (ANDA) mencionan que al Oeste del actual Lago se han encontrado fragmentos de figurillas y tiestos de cerámica debajo de dicha capa, al momento de la perforación de pozos de agua.

La zona arqueológica Chalchuapa, en donde se tiene registro de la ceniza de TBJ, es uno de los sitios arqueológicos de aquel entonces, abandonados a raíz de la erupción del Volcán Ilopango entre los Siglos V y VI d.C. (Sheets, 1983; Dull et.al., 2001; Dull, 2004; Dull et.al., 2010); no obstante, los datos obtenidos hasta la fecha en el área de Tazumal, una de las 10 áreas que conforman la zona arqueológica Chalchuapa hace reconsiderar la interpretación en mención.

En este artículo se revisará la estratigrafía de los sitios arqueológicos ubicados dentro del territorio salvadoreño, los cuales han registrado el estrato de la TBJ 


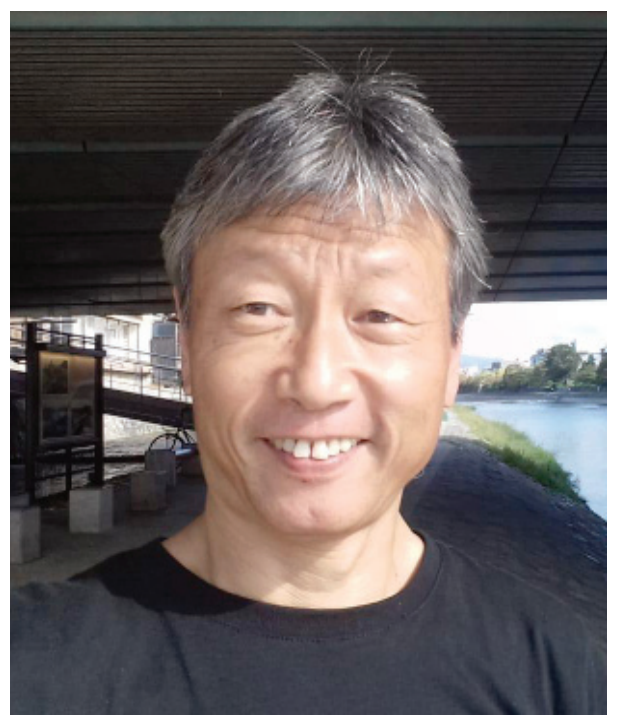

Shione Shibata

primaria, es decir la ceniza volcánica de color blanco caída directamente desde la caldera del Volcán Ilopango.

Se tomará como eje central, la observación de la estratigrafía posterior a la deposición de la TBJ primaria, debido a que la influencia de la erupción del Volcán Ilopango, se ha tratado en su mayoría, tomando como base los análisis físicos y químicos de la ceniza volcánica de dicha erupción y la distribución espacial de la misma junto con su grosor, es decir, desde un punto de vista geológico.

Por otro lado, se reconsiderará la manera en que afectó dicho evento, el área de Tazumal luego de la erupción tomando en cuenta la observación estratigráfica en diversos puntos en donde se han registrado las capas de TBJ primaria en la zona arqueológica Chalchuapa.

\section{Antecedentes de los estudios vulcanológicos sobre la TBJ}

Al principio del Siglo XX los habitantes de El Salvador habían llamado tradicionalmente la ceniza volcánica del color blanco situada alrededor del Lago de Ilopango como "Tierra Blanca" sin tomar en cuenta esta huella del evento natural que azotó la extensa área de Centroamérica.

El 7 de junio de 1917 el Volcán San Salvador conocido como El Boquerón levantó una gran capa de humo en el aire y su lava corrió en la falda Norte del 
mismo Volcán. El joven Jorge Lardé se impactó al ver este fenómeno natural y su interés se inclinó hacia el estudio de la vulcanología, sismología y arqueología (Viera Altamirano, 1951: 21).

Lardé hizo recorridos minuciosos en la Capital San Salvador y sus alrededores buscando capas de la ceniza volcánica del color blanco en diversos cortes geológicos con el fin de identificar el origen de dicha ceniza volcánica.

Finalmente Lardé concluyó su origen en la caldera de Ilopango la cual es el actual lago (Lardé, 1950: 78; Lardé, 1953: 82), ya que la ceniza volcánica del color blanco se observó por lo menos a 10 millas en todos los rumbos alrededor de Ilopango y no se registró dicha ceniza al Oeste del Volcán San Salvador (Lothrop, 1927: 171).

Al entrar en la década de los 1950's, geólogos de los Estados Unidos de Norteamérica y Alemania dieron inicio al estudio de la ceniza volcánica del color blanco, entre los cuales se pueden citar Howel Williams, Helmut MeyerAbich y Richard Weyl.

William y Meyer-Abich se refieren al origen del Lago de Ilopango y clasificaron preliminarmente el Lago de Ilopango no como una caldera sino como una "depresión volcano - tectónica" (Williams y Meyer-Abich, 1953: 7), mostrando el mapa de profundidad del lago de Ilopango, secciones diagramáticas para ilustrar el origen del mismo Lago y mapa geológico preliminar alrededor del Lago de Ilopango (ibid., 1953). En el mismo artículo se menciona el origen de la "Tierra Blanca", la cual proviene de la erupción del Volcán El Boquerón (ibid., 1953: 5 y 6).

Según las observaciones de la "Tierra Blanca" alrededor de la Capital San Salvador, las capas de ceniza y pómez se distinguen por lo menos 5 estratos, entre los cuales se registran las capas inferiores en los pocos sitios y las 3 superiores se observan en mayor área. Hay intervalo de calma en cada una de estas erupciones, los cuales pudieron ser largos, ya que se muestra descomposición en cada superficie. Debajo de la capa inferior se encuentra un estrato de humus que contiene materiales arqueológicos de hace 2000 años (ibid., 1953: 6).

Acerca de la distribución espacial de la "Tierra Blanca", a causa de los vientos del Oeste y Noroeste, se extiende hacia al Sur y Este del Volcán El Boquerón. Dichas cenizas de pómez blanco fueron lavadas y acumuladas en los valles ubicadas entre Santa Tecla y la Capital San Salvador (ibid., 1953: 6).

Por otro lado Weyl realizó los análisis de las cenizas de pómez llamadas "Tierra Blanca" y los fenómenos de descomposición de las cenizas de vidrio. Resultó 
que las capas de la "Tierra Blanca" procedente de las erupciones del Volcán Boquerón indicada por otros geólogos (Weyl, 1955).

En 1956 dentro del marco de las actividades del Servicio Geológico Nacional del Ministerio de Obras Públicas de El Salvador el Geólogo Helmut Meyer-Abich había realizado minuciosos estudios sobre los volcanes activos de los territorios nacionales de Guatemala y El Salvador, en los cuales se menciona que la ceniza volcánica denominada "Tierra Blanca joven" es originaria de las erupciones del Volcán Ilopango y su distribución espacial se extiende hacia el Este a las faldas del Volcán San Vicente, hacia el Oeste al valle de San Andrés, hacia el Sur a la planicie costera al Oriente de La Libertad y hacia el Norte aproximadamente a las faldas del Volcán Guazapa (Meyer-Abich, 1956: 69 y 76).

Meyer-Abich indica que una de las características de la "Tierra Blanca joven" es una capa delgada de cascajo respectivamente arena de pómez entre 1 a 5 $\mathrm{cm}$ de espesor, la cual se registra en todo su área de distribución. Debajo de dicha capa se encuentran materiales arqueológicos de la "cultura arcaica", cuyo fechamiento podría ser hace 2993 años con margen de error de \pm 360 años según el análisis ejecutado por W. F. Libby del Instituto para Estudios Nucleares en Chicago (ibid., 1956: 76).

Al final de la década de los 1960 's se logró otro avance del estudio sobre la "Tierra Blanca joven".

Durante los años de 1967 a 1971 la Misión Geológica Alemana había realizado los estudios geológicos y se elaboró el atlas geológico de El Salvador el cual consiste en 6 pliegos de mapa geológico (Weber et.al., 1974), sin embargo, no se encuentra la descripción detallada del atlas en mención y la "Tierra Blanca joven" podría ser el código "s4" del Holoceno, la cual es de piroclastitas acidas y epiclastitas volcánicas subordinadas (Comunicación personal con Shigeru Kitamura).

En el caso de que "s4" sea de los depósitos de materiales piroclástico, la Caldera de Ilopango está localizada en el centro del área de la distribución espacial de "s4", ya que se habían registrado materiales piroclásticos en el área "s4" indicada en el atlas geológico en mención durante el reconocimiento realizado por Kitamura (Comunicación personal con Shigeru Kitamura).

En el año 1975 Payson Sheets y Virginia Steen-McIntyre habían llevado a cabo el estudio general de la tefra de "Tierra Blanca Joven" en el área ubicada al Noroeste de El Salvador con la distancia más de $10 \mathrm{~km}$ desde el lago de Ilopango (Hart y Steen-McIntyre, 1983a: 15). 
La tefra de "Tierra Blanca Joven" se extiende en el área investigada y el tamaño de las partículas se hizo más grande hacia el Sureste por la dirección al Lago de Ilopango. En el corte estratigráfico se dividen 3 capas; la capa inferior es la ceniza gruesa, la media es la ceniza fina del color beige y la superior es la ceniza fina del color bronceado (ibid., 1983a: 15 y 16).

Desde el año 1978 hasta el 1980 Sheets dirigió el "Proyecto Protoclásico" en el valle de Zapotitán como el estudio de la Universidad de Colorado de los Estados Unidos de América. El interés particular de Sheets en esta expedición científica fue el efecto de la erupción del Volcán Ilopango ocurrida en el Siglo III d.C. (fechamiento tomado en aquel entonces basado en el análisis de C14) ante los habitantes del valle de Zapotitán (Sheets, 1983: 1 y 2).

Dentro del marco del Proyecto en mención William J. E. Hart y Virginia SteenMcIntyre realizaron los estudios vulcanológicos, especialmente de la erupción del Volcán Ilopango en el Siglo III d.C. En el campo los geólogos recorrieron diversas partes del territorio nacional de El Salvador en el año de 1978. Hacia el Este, Noreste y Sureste del Lago de Ilopango se hicieron observaciones sobre los cortes estratigráficos de 16 sitios y hacia el Oeste, Noroeste y Suroeste de la misma caldera se registraron las huellas de TBJ en 34 sitios, con el fin de recopilar datos de la tefra de TBJ (Hart y Steen-McIntyre, 1983a: 16-19).

A consecuencia de los trabajos de campo se pudo comprender que la tefra de TBJ consiste en 6 unidades; desde la inferior a la superior, (1) Ceniza fina en la base, (2) Ceniza gruesa basal, (3) T2: depósito de caída, (4) Unidad oleada piroclástico, (5) T1: flujo de ceniza, (6) T1: depósito de caída (ibid., 1983a:19-20; Kitamura, 2017:45). Además se indica 4 tipos de mapa de distribución espacial de TBJ, los cuales son (A) diámetro en promedio ( $\mathrm{mm}$ ) de los fragmentos de 5 pómez (ceniza gruesa basal) más grandes recolectados de diferentes sitios, (B) grosor de T2: depósito de caída, (C) grosor de T1: flujo de ceniza y T1: depósito de caída, (D) grosor preservado de la tefra de TBJ (Hart y Steen-McIntyre, 1983a: 21 y 22).

En el laboratorio de la sede de campo y el de Steen-McIntyre en Colorado se realizaron los análisis físicos - químicos de las muestras de la tefra de TBJ (ibid., 1983a: 19, 22-26).

Con base en las evidencias de los trabajos de campo y laboratorio, se concluyó lo siguiente: la erupción del Volcán Ilopango que produjo la tefra de TBJ pudo ser de grandes magnitudes y de forma violenta. Dicho evento volcánico pudo durar corto tiempo; sin embargo, los depósitos de la ceniza se extendieron ampliamente en la región Centroamericana (ibid., 1983a: 26). 


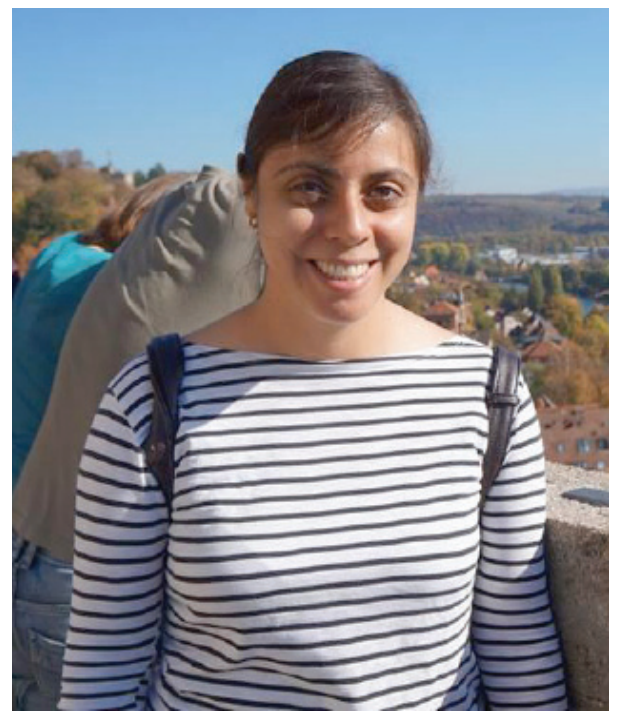

Rocío Herrera

Por tal situación los habitantes dentro de un radio de $25 \mathrm{~km}$ desde el Lago de Ilopango murieron inmediatamente y los pobladores del área un poco más lejos del radio de $25 \mathrm{~km}$ desde el mismo Lago se enfrentaron con una muerte lenta a consecuencia de gases venenosos, quemaduras y escaldaduras causadas por la caída de la ceniza de T2. Las personas que vivían en el área más alejada del Volcán Ilopango sufrieron la contaminación de alimentos, agua y aire. Es muy probable que el efecto de la erupción del Volcán Ilopango del Siglo III d.C. se extendió a cientos o miles de kilómetros a favor del viento en forma de lluvia acida que mataría los cultivos y lodo fijado con fosfato (ibid., 1983a: 30).

Esta interpretación acerca del efecto de la erupción del Volcán Ilopango en el Siglo III d.C. fue bastante aceptada; sin embargo, sobre el fechamiento de dicha erupción se presentaron posteriormente los otros datos con base a los análisis de las muestras recolectadas de nuevo, utilizando el método de la espectrometría de masas con aceleradores en la datación por radio carbono (AMS por sus siglas en inglés). Con este método Robert A. Dull hizo la reconsideración del fechamiento de la erupción de la TBJ en base a los análisis de las muestras recolectadas sobre la carretera que de San Salvador conduce a Santa Ana ubicado a $2 \mathrm{~km}$ hacia el Nornoroeste de Santa Tecla, en el mes abril del año 1998. Según la nueva datación, la erupción del Volcán Ilopango podría ser en 1 sigma: 421 (429) 526 años d.C. y 2 sigma: 408 (429) 536 años d.C. (Dull et.al., 2001: 25, 27-30).

Además, por la revisión de los sitios arqueológicos, la cerámica y la datación de diversos sitios arqueológicos Dull concluyó que el efecto de la erupción de 
la TBJ provocó un colapso demográfico a gran escala en el área sufrida por la mayor devastación tales como en el valle de San Salvador y la parte Este del valle de Zapotitán, el Occidente de El Salvador y el Sureste de Guatemala (ibid., 2001: 25).

En el valle de San Salvador y la franja Este del valle de Zapotitán la mayoría de los habitantes deben haber perecido inmediatamente por el flujo piroclástico y la gruesa capa de depósito caído desde el Volcán Ilopango (ibid., 2001: 39).

Alrededor de la cuenca del Río Paz y en el área costeña Suroeste de El Salvador los pobladores probablemente abandonaron sus lugares originarios a causa de la evacuación inmediata, el hambre por el colapso agrícola, las enfermedades y el colapso de la economía regional provocados por dicha erupción (ibid., 2001: $39-40)$.

En el Sureste de Guatemala la erupción de la TBJ no parece haber tenido efectos biofísicos severos. El decremento en población se debió a la desarticulación de la red económico-cultura de la esfera Miraflores (ibid., 2001: 40).

Los habitantes sufrieron a causa de la erupción del Volcán Ilopango y migraron hacia el Noroeste y el Norte, lo que derivó en el crecimiento de la población rural del valle de Guatemala y del centro urbano de Copán, Honduras (ibid., 2001: 40).

El tiempo de recuperación de las áreas afectadas de la erupción de TBJ en El Salvador y el Sureste de Guatemala puede llegar a estimarse hasta el Siglo VII d.C. (ibid., 2001: 25).

Dull realizó estudios estratigráficos de sedimentos en 4 lugares en el Occidente de El Salvador: Laguna Cuzcachapa, Laguna El Trapiche (ambas ubicadas en la zona arqueológica Chalchuapa), Laguna Llano (cerca de la ciudad de Ahuachapán, en la cuenca del Río Paz) y Laguna Verde (localizada en la Sierra Apaneca).

El objetivo de los estudios en mención fue conocer el ambiente natural antes y después de la erupción de TBJ del Volcán Ilopango en la cuenca del Río Paz y la Sierra de Apaneca (Dull, 2004: 238).

El grosor acerca de los depósitos de la tefra de TBJ en el Occidente de El Salvador varía entre 20 y $50 \mathrm{~cm}$ en las áreas terrestres y desde 15 a $25 \mathrm{~cm}$ en las lagunas (Ibid., 2004: 242). 
La ecología del Occidente de El Salvador antes de la erupción del Volcán Ilopango estaba muy marcada por el uso intensivo de terrenos, extensos cultivos, deforestación, quema de terrenos y bajada del nivel de agua en las lagunas (ibid., 2004: 242).

El medio ambiente natural del mismo Occidente después de la caída de la TBJ, subió el nivel de agua en las lagunas de Llano, El Trapiche y Verde. Los impactos a la producción agrícola aparecen muy severos, lo cual provocaría la emigración a otras regiones no afectadas por este evento volcánico. Chalchuapa fue aparentemente abandonado después de la erupción en base a los datos sobre la falta del polen Zea en la capa inmediata sobre la de TBJ en los sedimentos lacustres. No es claro el tiempo del abandono en la zona arqueológica Chalchuapa; sin embargo, la organización política del nivel cacicazgo pudo ser reconstituida antes del final del Siglo VI d.C. (ibid., 2004: 243).

Según los datos de sedimentos recolectados en Laguna Verde de la Sierra de Apaneca es notorio el abandono total del asentamiento agrícola en dicha área (ibid., 2004: 243).

El registro del polen en Laguna Llano ubicada en Ahuachapán no indica claramente el hiato de la ocupación humana (ibid., 2004: 243).

En el año 2010 Dull presentó el fechamiento nuevo de la erupción de TBJ del Volcán Ilopango junto con otros investigadores en base al análisis de las 18 nuevas muestras de carbón. En la presentación se menciona que el evento de carga del aerosol atmosférico más grande ocurrió en el año ca. 536 años d.C., la cual fue una niebla seca que envolvió gran parte de la esfera terrestre, seguida por un enfriamiento global prolongado, la cual habría durado desde ca. 536 años d.C. hasta ca. 550 años d.C. Según registros de anillos de crecimiento de árboles, cuentas históricas y datos arqueológicos, los efectos aparecieron en el Hemisferio Norte, en donde las temperaturas frías en el verano y las malas cosechas afectaron las sociedades, desde China hasta el Mediterráneo y Mesoamérica (Dull et.al., 2010).

Una revisión sobre las cronologías del núcleo de hielo y los registros de deposición de sulfato volcánico $\left(\mathrm{SO}_{4}\right)$ recolectados en las áreas de la Antártida (LMD) y Groenlandia (Dye-3, GRIP y NGRIP) refiere a una gran erupción volcánica en la región tropical como fuente probable. Los datos acerca de la erupción de TBJ del Volcán Ilopango son consistentes con el evento arriba mencionado en términos de cronología, magnitud y ubicación geográfica (ibid., 2010). 
En la misma presentación se menciona que se registró la gruesa capa de la tefra de TBJ en el Oriente de El Salvador y según los estudios realizados por Steffen Kutterolf y Armin Freundt la trayectoria de la erupción de TBJ se dirigiría hacia el Sur y Sureste (ibid., 2010).

El Proyecto Arqueológico de El Salvador comenzó sus investigaciones en la Trinchera $4 \mathrm{~N}$ del Parque Arqueológico Casa Blanca de la zona arqueológica Chalchuapa desde el mes de septiembre del año 2000, bajo la dirección de Nobuyuki Ito, profesor asistente de la Universidad de Nagoya, Japón (Ito, 2010).

Dentro del mismo Proyecto Shigeru Kitamura, profesor asociado de la Universidad de Hirosaki-Gakuin se encargó la sección del estudio de vulcanología. Kitamura había realizado los estudios de la geomorfología volcánica y la estratigrafía de tefra en el área que abarca desde el Volcán El Chingo hasta la Sierra de Apaneca (Kirtamura, 2010: 249-256).

El Proyecto Arqueológico de El Salvador continuó sus investigaciones desde el mes agosto de 2004, cambiando el campo de estudio hacia el área de Tazumal de la misma zona arqueológica (Ito, 2016).

Durante el tiempo de este Proyecto Kitamura llevó a cabo los estudios estratigráficos de la tefra originaria de diversos volcanes que se encuentran en el Occidente de El Salvador, especialmente alrededor de la zona arqueológica Chalchuapa (Kitamura, 2016a: 300-343).

Según observaciones sobre las 4 capas de tierra blanca en la Trinchera $4 \mathrm{~N}$ del Parque Arqueológico Casa Blanca, el vulcanólogo japonés determinó que las 3 capas superiores redepositadas de tierra blanca que miden aproximadamente entre 40 y $50 \mathrm{~cm}$ de grosor son la tefra secundaria de TBJ (Kitamura, 2001:54; Kitamura, 2016a: 308) y la capa inferior que oscila entre 16 y $18 \mathrm{~cm}$ de espesor es la tefra de TBJ primaria (Kitamura, 2010: 252; Kitamura, 2016a: 308).

Kitamura verificó los afloramientos de depósitos naturales en la ladera y la falda del Volcán Santa Ana, los cuales son originarios de la tefra de TBJ primaria, cuyo grosor mide entre 12 y 14 cm (Kitamura, 2010; Kitamura, 2016a: 308).

Con base en estos resultados y al reconsiderar los datos acerca de la tefra de TBJ registrados en Chalchuapa los cuales fueron presentados por los estudios anteriores; uno procedente del área pantanosa de la Laguna Seca en donde se registraron las capas de la tefra de TBJ con un grosor promedio de $50 \mathrm{~cm}$ (Hart et.al., 1983b: 42) y otro proveniente del sedimento lacustre de la Laguna Cuzcachapa cuyo espesor mide $25 \mathrm{~cm}$ (Dull, 2004: 239), Kitamura concluyó que el espesor de $50 \mathrm{~cm}$ de la tefra TBJ primaria en la zona arqueológica 
Chalchuapa estaría sobrevalorado y es necesario realizar una revisión de ese dato en el área de cobertura de la tefra de TBJ presentado anteriormente (Kitamura, 2016a: 308).

En el informe preliminar del Proyecto Arqueológico de El Trapiche, Chalchuapa, el Vulcanólogo Japonés presentó el estudio más reciente sobre la tefra de TBJ, donde se muestra gráficamente el supuesto límite del flujo de pómez de TBJ (Kitamura, 2017: 46), el cual alcanza al Oeste del Lago de Ilopango en la parte Este del Valle de Zapotitán y la estimación de la caída de TBJ podría ser menos que la considerada por los investigadores anteriores (Fig. 2 y 3 ).

\section{Figura 2}

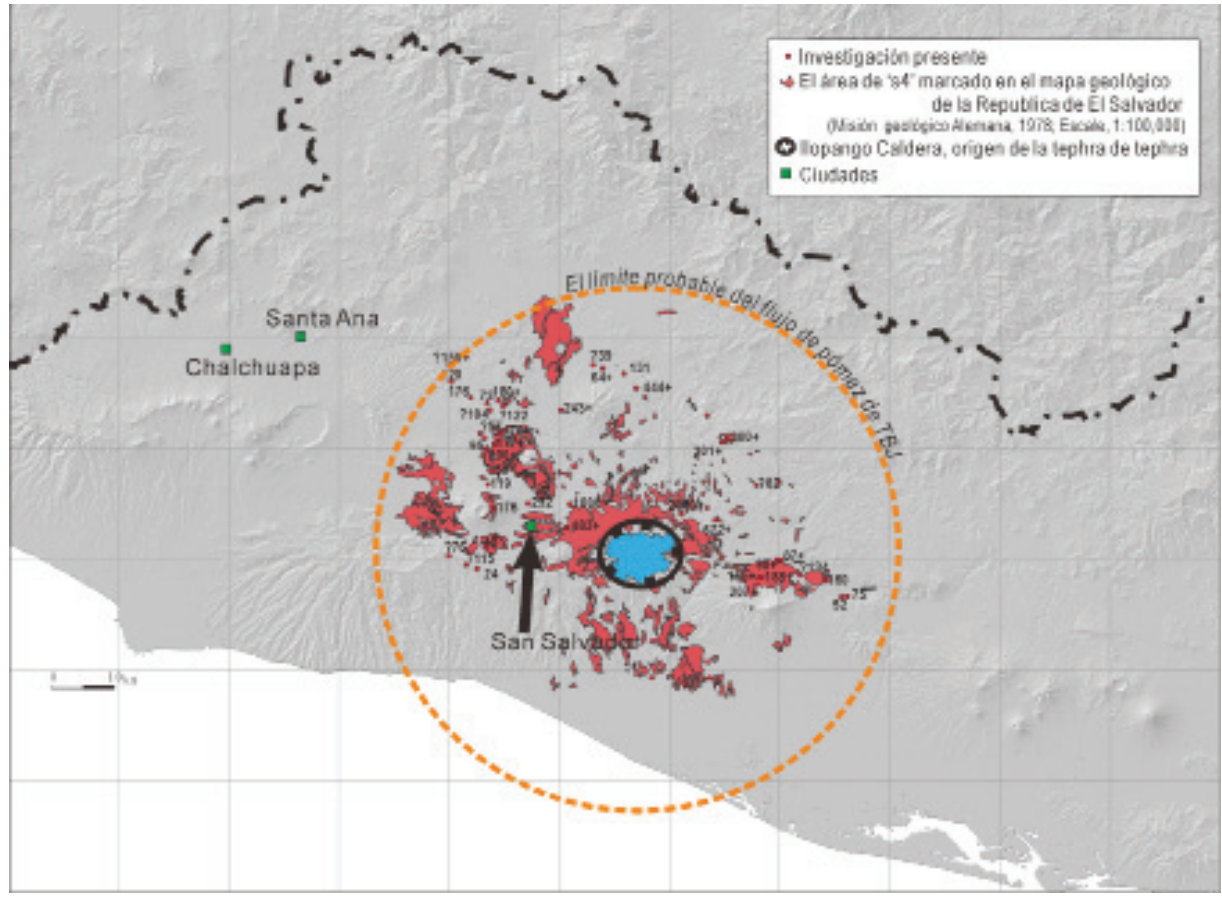

Distribución espacial del flujo de pómez y caída de la TBJ (Área emplazada del flujo de pómez de la TBJ) (cortesía de Shigeru Kitamura). 


\section{Figura 3}

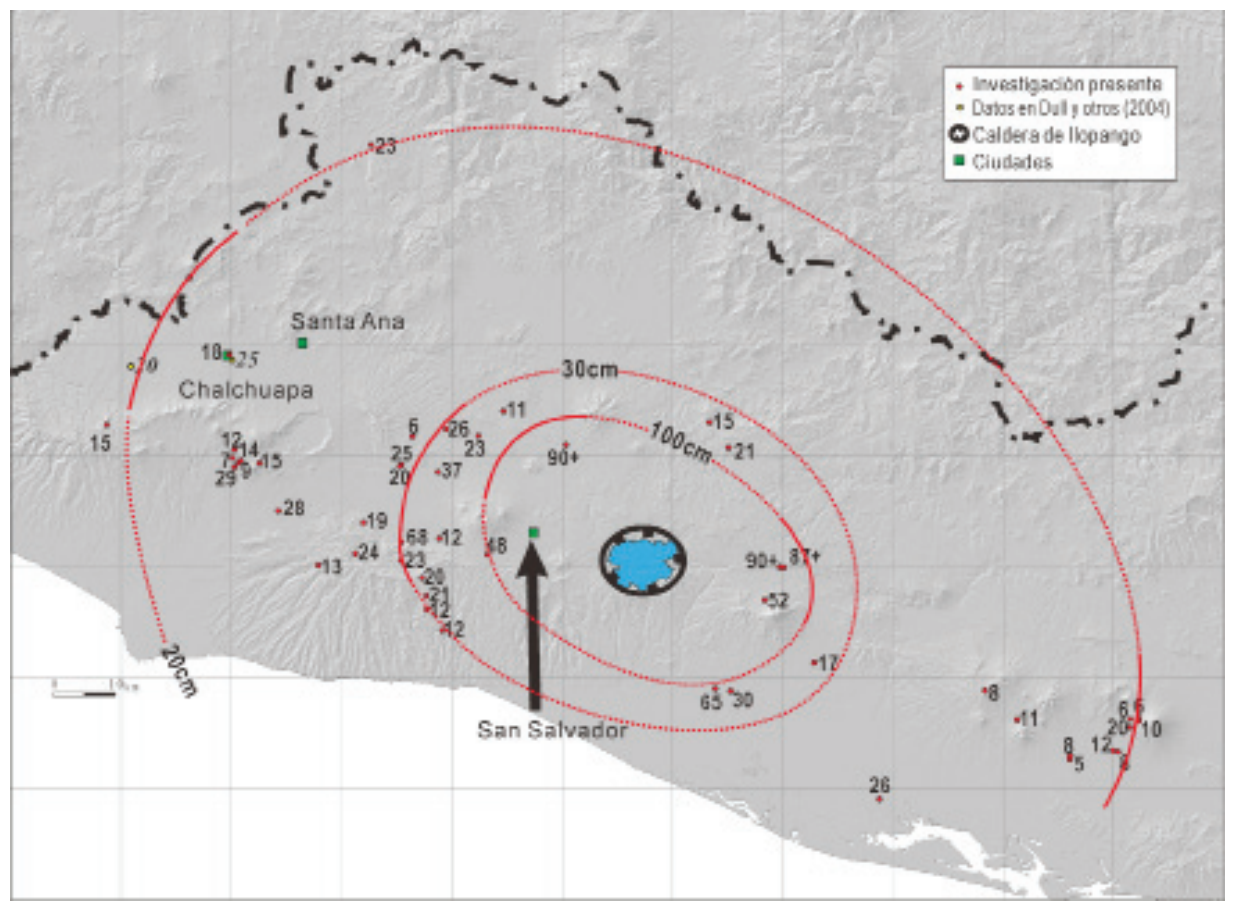

Distribución espacial del flujo de pómez y caída de la TBJ (Distribución de la caída de la TBJ) (cortesía de Shigeru Kitamura).

Kitamura cita la estratigrafía de TBJ presentada por el Geólogo Walter Hernández, quien divide más detalladamente las unidades de la tefra de TBJ (Hernández, 2004; Kitamura, 2017: Figura 25).

\section{Sitios arqueológicos con presencia de TBJ primaria registrados en El Salvador}

En el territorio salvadoreño existen más de 671 sitios arqueológicos registrados en el atlas arqueológico, dentro de los cuales hay asentamientos prehispánicos con huellas de la TBJ primaria.

En este capítulo se analizará respectivamente la estratigrafía de dichos sitios arqueológicos por las áreas geográficas que se dividen en (1) San Salvador y su contorno, (2) Valle de Zapotitán, (3) Norte de San Salvador y (4) Sureste del Lago de Ilopango. 


\section{San Salvador y su contorno}

Figura 4

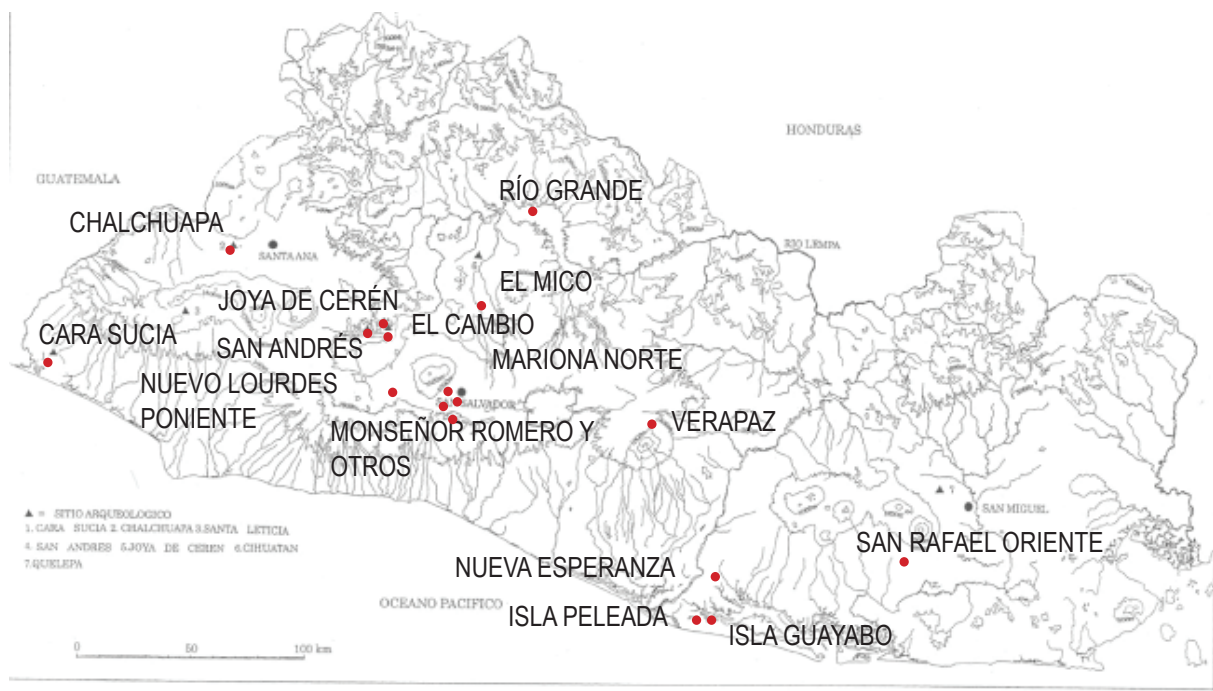

Ubicación de los sitios arqueológicos en donde está registrada la TBJ primaria.

Los reconocimientos realizados por Jorge Lardé, quien junto con Samuel K. Lothrop seleccionó el lugar ubicado entre Cerro de El Zapote y el Río Acelhuate en la zona de San Jacinto, San Salvador, en donde se llevó a cabo una excavación desde el mes enero de 1926, utilizando primera vez en El Salvador el método de estratigrafía (Lothrop, 1927: 168-173). Resultó que se confirmaron respectivamente 2 capas culturales de la época prehispánica sobre la capa de TBJ y debajo de la misma (ibid., 1927: 173-187).

Boggs menciona la capa de la ceniza volcánica de color blanco denominada como Tierra Blanca por habitantes en el artículo acerca del rescate arqueológico realizado en el año 1946 sobre la Loma de Tacuazín ubicada en la Finca Belén, San Salvador (Boggs, 1966: 178-179). Cabe mencionar que Boggs pensaba en el principio dicha ceniza volcánica del color blanco procedente de la erupción del Volcán San Salvador (ibid., 1966: 183).

Desde la década de los 1980's se tiene un crecimiento acelerado de la urbanización en el área capitalina de San Salvador (Herodier, 1997: 49) y en los cortes de la construcción de carreteras, calles, lotificaciones, entre otros se han registrado surcos de cultivo sepultados debajo de la TBJ primaria, los cuales 
fueron registrados en las áreas de San Salvador, Antiguo Cuscatlán, Cumbres de Cuscatlán y Santa Tecla (Amaroli et.al., 1999: 566). En esos lugares de hallazgos no se observó la ocupación posterior o se registró la ocupación del Período Clásico Tardío y/o Postclásico.

Desde el inicio del Siglo XXI hasta la fecha aumentaron los números de hallazgos sobre surcos de cultivo en el área fronteriza entre los municipios de San Salvador, Antiguo Cuscatlán y Santa Tecla. En el mes junio del año 2007 se encontraron más de 70 surcos de cultivo debajo de la capa de la TBJ primaria a más de $5 \mathrm{~m}$ de grosor, observados en ambos paredones de un corte de calle en el boulevard Monseñor Romero al Norte del centro comercial Multi Plaza

\section{Fotografia 2}

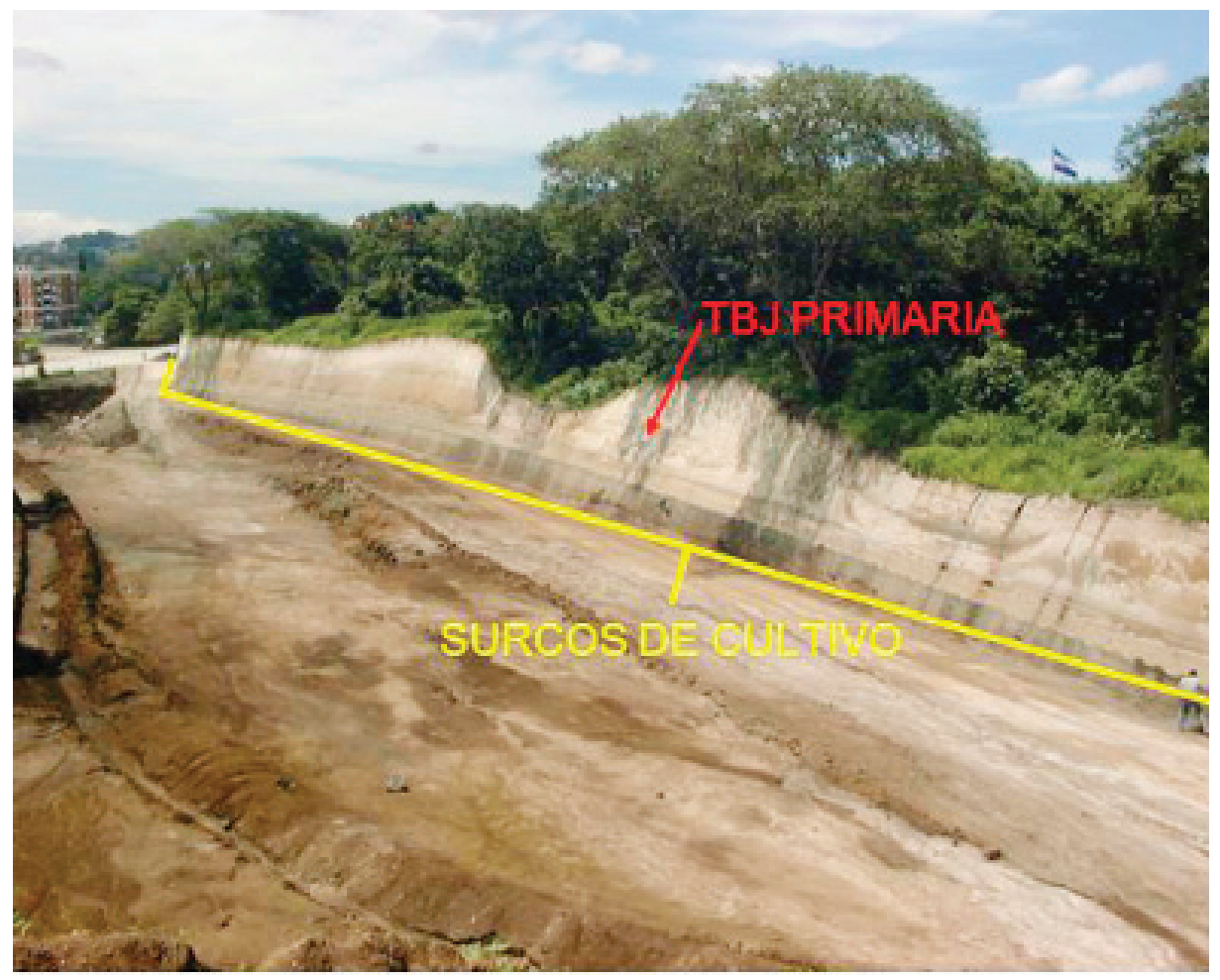

Surcos de cultivo encontrados debajo de la TBJ primaria sobre el Boulevard Monseñor Romero.

En el área residencial de la Colonia San Benito se registraron por lo menos 3 sitios de surcos de cultivo sepultados por la misma ceniza volcánica. Desde el punto de vista estratigráfico, en los campos de cultivo arriba mencionados no se observó la ocupación posterior a la época prehispánica. 
Por otro lado en diversos lugares del centro de San Salvador se han registrado materiales arqueológicos del Período Clásico Tardío y Postclásico encima de la TBJ secundaria, debajo de la cual se encuentra la capa de la TBJ primaria, cuyo grosor podría ser más de $15 \mathrm{~m}$, aumentando mucho más hacia el rumbo del Lago de Ilopango.

Al analizar los contextos posteriores de la caída de la TBJ en el área de San Salvador y su contorno, el Valle de Las Hamacas fue abandonado totalmente a causa de la erupción del Volcán Ilopango entre el Siglo V y VI. La reocupación del mismo Valle pudo esperar hasta el Período Clásico Tardío $(600-900$ años d.C.).

\section{Valle de Zapotitán}

Figura 4

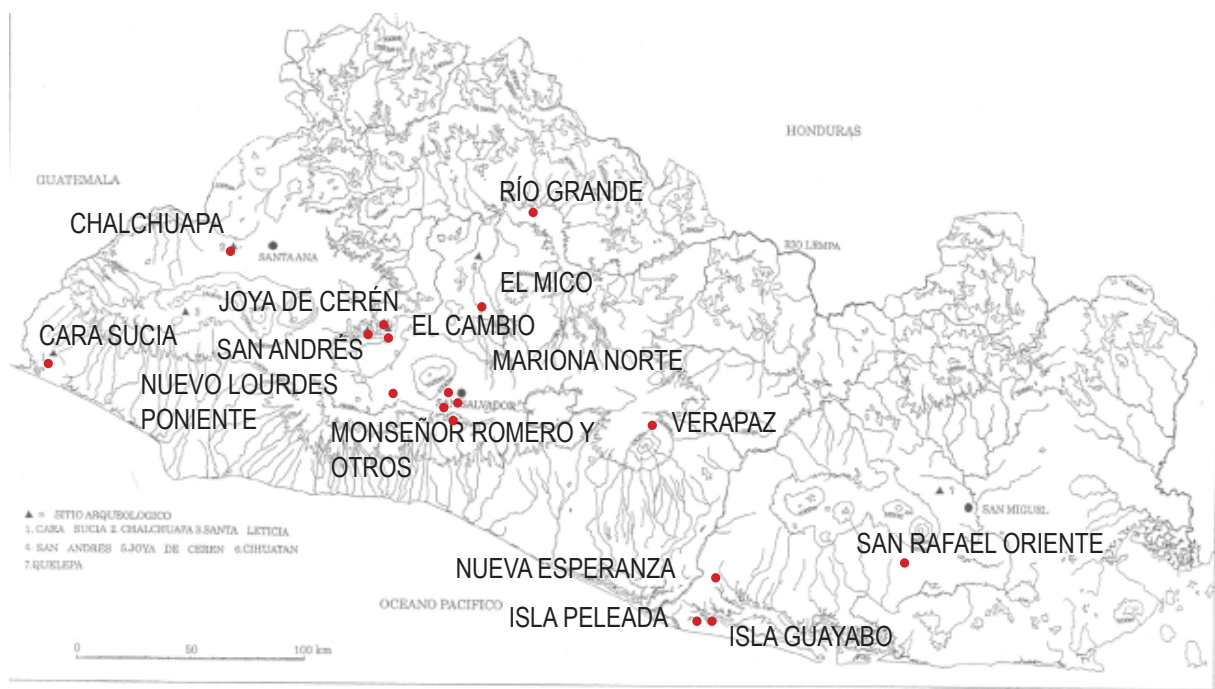

Ubicación de los sitios arqueológicos en donde está registrada la TBJ primaria.

El Valle de Zapotitán está ubicado aproximadamente a $35 \mathrm{~km}$ al Oeste del Lago de Ilopango. En dicho Valle están registrados los sitios arqueológicos del Período Preclásico Tardío, por ejemplo, Nuevo Lourdes Poniente, San Andrés y El Cambio.

El sitio arqueológico Nuevo Lourdes Poniente está ubicado sobre la topografía fluvial formada por el Río Los Choros en la orilla Sureste del Valle de Zapotitán, 
en donde se había realizado los rescates y sondeos arqueológicos entre los años de 2013 y 2014.

En dicho sitio se registró debajo de la TBJ primaria un campo de cultivo inmenso con surcos, cuya dimensión mide por lo menos $488,800.00 \mathrm{~m}^{2}$; sin embargo, dejando la capa de la tierra del color café-negra con aproximadamente $20 \mathrm{~cm}$ de grosor la reocupación del sitio comenzaría desde el Período Clásico Tardío (Comunicación personal con Hugo Díaz, Julio Alvarado y Michelle Toledo; Morán, 2013; Ramos, 2018).

Los orígenes del Río Sucio se encuentran en el Oeste y Sur del Valle de Zapotitán y a lo largo de la cuenca media del Río Sucio se encuentran los sitios arqueológicos San Andrés y El Cambio.

En el sitio arqueológico San Andrés hay huellas de la ocupación del Período Preclásico Tardío; sin embargo, no se han encontrado basamentos piramidales del mismo período, dejando la posibilidad de la existencia de subestructuras en los interiores de los montículos ubicados en La Acrópolis y La Campana (Boggs, 1943; Beagley et.al., 1996; Begley et.al., 1997; Ichikawa et.al., 2016). En el pozo denominado Fosa N79-80 12-13W por Boggs, ubicado al Norte de la Estructura 1 de La Acrópolis se indica la existencia de una capa de la ceniza volcánica, supuestamente TBJ primaria, dejando una capa de tierra con aproximadamente $50 \mathrm{~cm}$ de grosor, sobre la cual se colocaron adobes y se colocó un piso de argamasa (Boggs, 1943: Perfil compuesto de Plaza Sur - Parte Sur y Montículo 1 - Parte Norte).

En la Estructura 5 denominada como La Campana se registró una capa de la TBJ primaria. Arriba de la capa de la TBJ secundaria dejando $15 \mathrm{~cm}$ aproximadamente un suelo del color café-negro se construyó una estructura hecha de piedra cortada, sobre la cual se edificaron las estructuras repelladas de argamasa con núcleo de adobe y tierra. Según el análisis de cerámica, dichos basamentos piramidales pertenecen al Período Clásico Tardío (Comunicación personal con Akira Ichikawa).

En el sitio arqueológico El Cambio se han registrado surcos de cultivo alrededor del montículo principal hecho de tierra, los cuales fueron cubiertos por la TBJ primaria, no obstante, la reocupación humana posterior a la caída o deposición de TBJ primaria pudo esperar hasta el Período Clásico Tardío (Shibata et.al., 2009).

Al analizar la estratigrafía de los sitios arqueológicos Nuevo Lourdes Poniente, San Andrés y El Cambio, el área Este del Valle de Zapotitán, al igual que el Valle de Las Hamacas, fue abandonado después de la caída de la TBJ primaria y 
su recuperación por grupos humanos se pudo extender hasta el Período Clásico Tardío o por lo menos hasta 600 años d.C.

Cabe mencionar que el sitio arqueológico Joya de Cerén, una aldea de la cultura maya sepultada por la erupción del Volcán Loma Caldera aproximadamente durante la primera mitad del Siglo VII (Sheets, 2013: 66), se encuentra estratigráficamente arriba de la TBJ primaria y se observa una capa de tierra de color café intercalada entre la TBJ y el nivel de ocupación de la aldea prehispánica Joya de Cerén (ibid., 2013: Figura 1-6). Es decir que dicha población se formó cierto tiempo después de la erupción del Volcán Ilopango (ibid., 2013: 31 y 32).

\section{Norte de San Salvador}

\section{Figura 4}

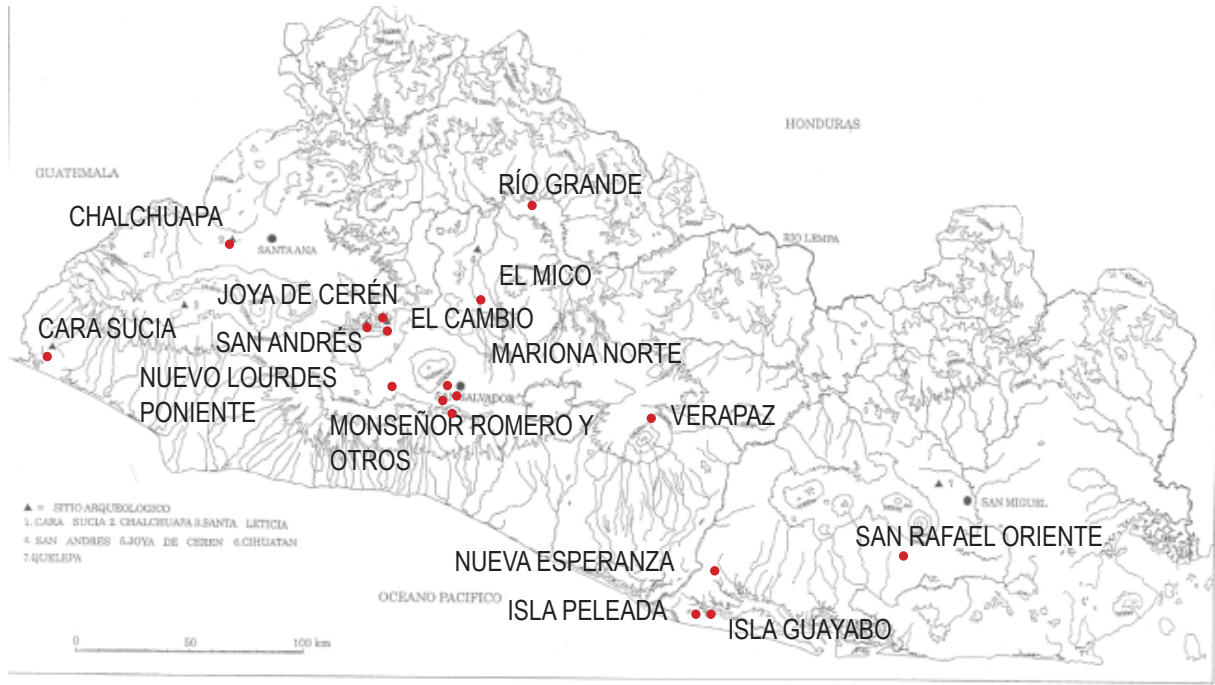

Ubicación de los sitios arqueológicos en donde está registrada la TBJ primaria.

El sitio arqueológico Cerro El Carmen se encuentra a $8 \mathrm{~km}$ al Noreste del Lago de Ilopango. En dicho lugar se registró un entierro primario debajo de la TBJ; sin embargo no se observaron huellas de la actividad humana sobre la capa de la misma ceniza volcánica con el grosor de $2.8 \mathrm{~m}$ (Erquicia et.al., 2016: 561-566).

El sitio arqueológico Mariona Norte está ubicado al Norte del centro penal. En una vivienda moderna ubicada en cantón Los Llanitos se perforó una fosa séptica y se encontró a aproximadamente $6 \mathrm{~m}$ de la profundidad una figurilla 
típica del Período Preclásico Tardío. Al revisar el corte estratigráfico en la calle de acceso ubicado al Norte de la misma vivienda se observó la capa de la TBJ primaria con el grosor más de $6 \mathrm{~m}$.

A unos $100 \mathrm{~m}$ de distancia hacia el Este de la vivienda en mención se encontró una formación troncocónica. Dicha fosa estaba rellenada con tierra y su boca estaba cubierta con la gruesa capa de la TBJ primaria. Es decir que la formación troncocónica fue abandonada antes de la erupción del Volcán Ilopango. Las vasijas encontradas dentro de la formación troncocónica pertenecen al Período Preclásico Medio y Tardío Temprano según su estilo (Méndez et.al., 2009: 1828). Posterior al evento volcánico de Ilopango, la zona no volvió ser ocupada.

El sitio arqueológico El Mico está ubicado a $2 \mathrm{~km}$ al Norte del centro de la ciudad de Guazapa y al Norte del Río Guazapa. Según la ficha del atlas arqueológico que posee la Dirección de Arqueología del Ministerio de Cultura, se han registrado diversas plataformas y terrazas. Hay 2 plataformas relativamente grandes que están conectadas por una terraza. Este sitio pertenece al Período Clásico según los tiestos de cerámica.

En la descripción sobre "Tierra del sitio", se mencionan humus y ceniza volcánica. Por lo tanto, las estructuras arriba mencionadas estarían construidas sobre la capa de ceniza volcánica.

A consecuencia del reconocimiento del sitio, la ceniza volcánica indicada en la ficha es originaria de la erupción del Volcán Ilopango entre el Siglo V y VI d.C., en donde está sepultado un bosque de aquel entonces por el flujo piroclástico (Kitamura et.al., 2007:55).

Cabe mencionar que la distancia entre la caldera del Volcán Ilopango hasta El sitio arqueológico El Mico es de aproximadamente $25 \mathrm{~km}$.

A unos $43 \mathrm{~km}$ al Norte de la Caldera Ilopango se encuentra el sitio arqueológico Río Grande en el valle de Paraíso. Este sitio es uno de los sitios sumergidos por entrar en la operación de la presa hidroeléctrica Cerrón Grande en 1976, en el cual se descubrieron surcos de cultivo sepultados debajo de la TBJ primaria. El artículo no menciona si se registró ocupación inmediata después de la caída de la TBJ (Earnest, 1976:64-66).

Al observar los sitios arqueológicos Mariona Norte, El Mico y Río Grande ubicados al Norte de San Salvador, no hay indicio de ocupación humana después de la caída de la TBJ primaria. 


\section{Sureste del Lago de Ilopango}

Figura 4

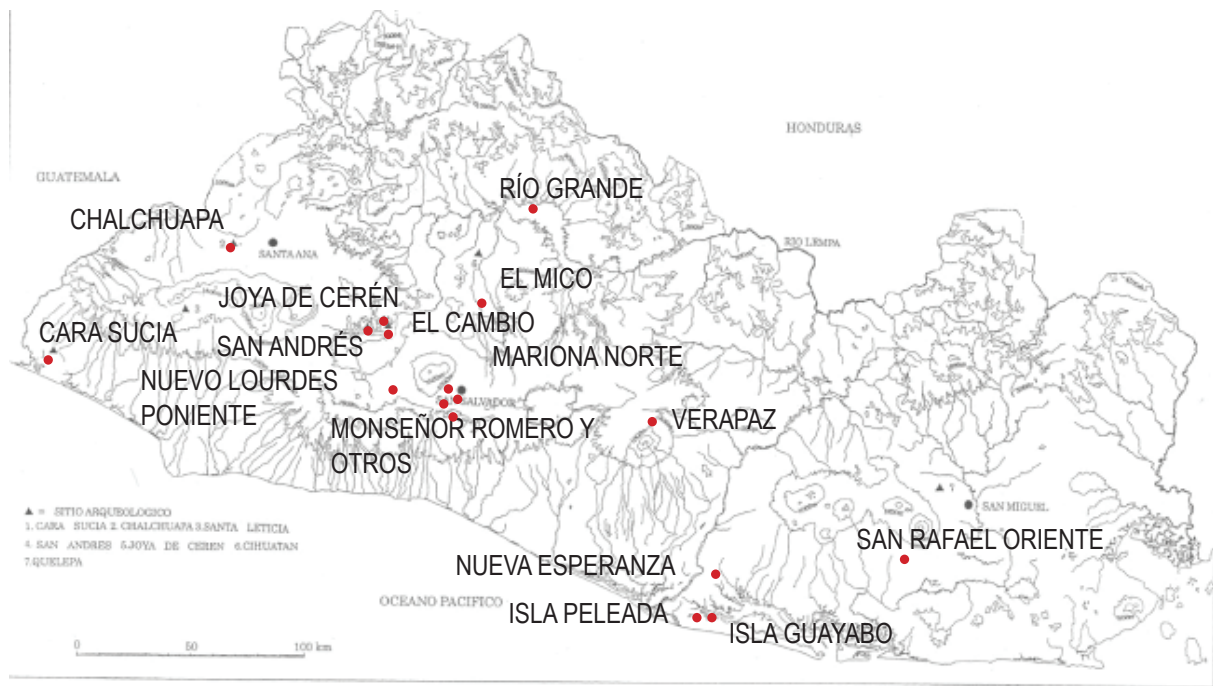

Ubicación de los sitios arqueológicos en donde está registrada la TBJ primaria.

El sitio arqueológico Nueva Esperanza se encuentra debajo de la Comunidad del mismo nombre, cuyos habitantes son repatriados desde León, Nicaragua en 1991 a causa de la guerra civil ocurrida en El Salvador entre 1980 y 1992. Dicho sitio está ubicado geográficamente en el área fluvial del Río Lempa, aproximadamente a $16 \mathrm{~km}$ desde la bocana del mismo Río.

En el sector Sur de la Comunidad Nueva Esperanza comenzaron las excavaciones en diciembre de 2007 a consecuencia de un hallazgo fortuito y hasta el año 2014 se realizaron 3 temporadas de proyecto arqueológico. Los resultados de los estudios arqueológicos en Nueva Esperanza, indican que la población prehispánica de la zona dependía de la producción de sal y enterraban a sus muertos con ofrendas de vasijas, entre otros (Ichikawa, 2011 y 2015).

Por la erupción del Volcán Ilopango entre el Siglo V y VI, se depositó la ceniza volcánica, la cual se acumuló en unos $35 \mathrm{~cm}$ de grosor, en segundo lugar, azotó una inundación al menos 10 veces seguidas (Comunicación personal con Shigeru Kitamura; Kitamura 2016b: 257) y el sitio fue sepultado totalmente debajo de la TBJ secundaria con un grosor aproximado de $1.2 \mathrm{~m}$. No se registró la reocupación en dicha área hasta la época republicana. 


\section{Fotografia 3}

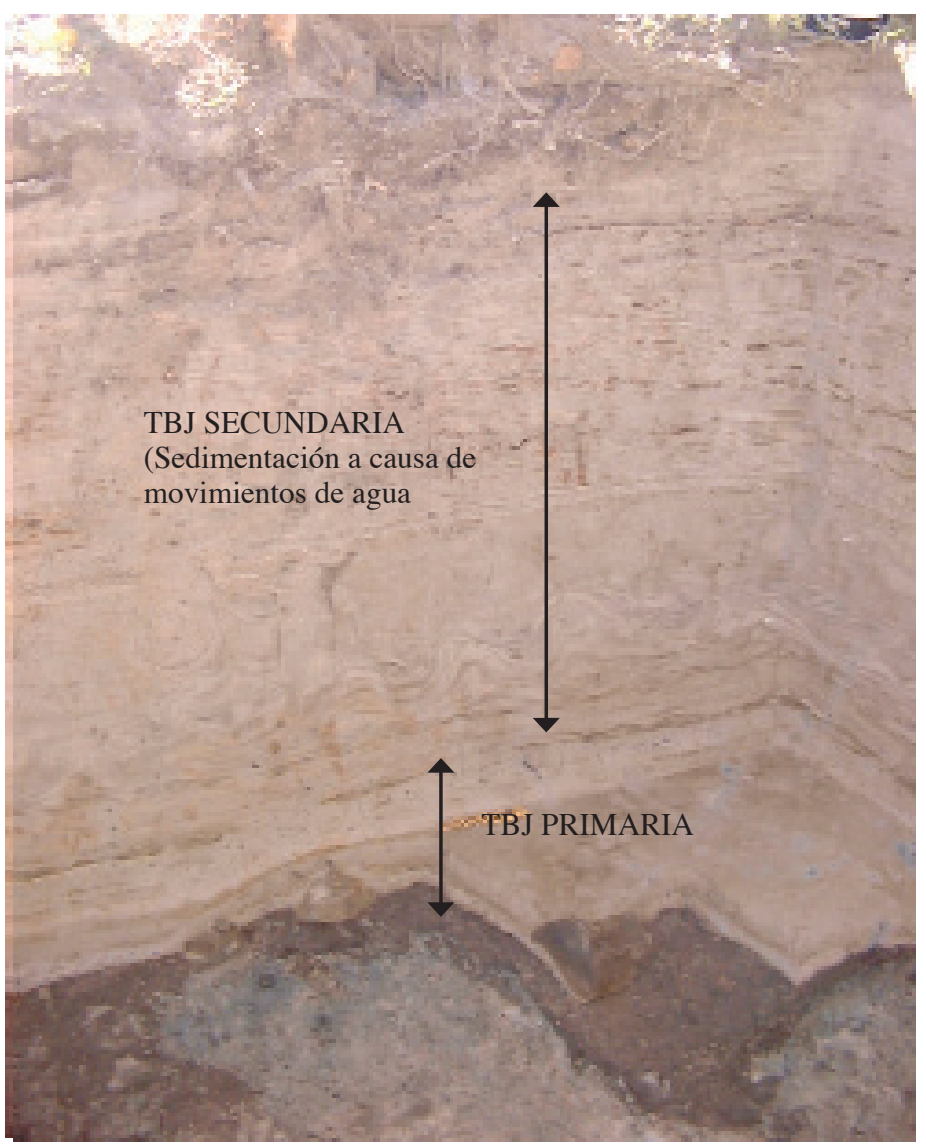

Capas de la TBJ primaria y secundaria observadas en el sitio arqueológico Nueva Esperanza Sector Sur.

Dentro de la Bahía de Jiquilisco se encuentran islas, entre las cuales la Isla Peleada y la Isla Guayabo están registradas debido a la alta cantidad de tiestos debajo de la TBJ primaria; sin embargo, no se observa estratigráficamente la ocupación del período posterior (Comunicación personal con Michelle Toledo y Shigeru Kitamura; Kitamura, 2016b).

El Departamento de Arqueología del entonces CONCULTURA recibió un aviso ciudadano de un hallazgo fortuito en el final del mes agosto de 2005 en el municipio de San Rafael Oriente, departamento de San Miguel, en donde se encontró un entierro sedente con ofrenda de 2 vasijas en un terreno en proceso de lotificación. 
Dicho entierro no se pudo ubicar estratigráficamente a causa de la remoción de tierra; sin embargo, por el estilo de las 2 vasijas, la osamenta podría pertenecer al Período Preclásico Medio. En el corte estratigráfico registrado en la lotificación se observó una capa identificada como TBJ primaria. En este sitio tampoco se registró una ocupación inmediata posterior a la erupción de la Caldera Ilopango.

El sitio arqueológico Quelepa es uno de los grandes centros ceremoniales de la época prehispánica en El Salvador y está ubicado aproximadamente a $95 \mathrm{~km}$ al Sureste desde la Caldera de Ilopango.

La ocupación humana del sitio arqueológico en mención comenzó desde el Período Preclásico Medio Tardío o Preclásico Tardío y continuó sin interrupción hasta el final del Clásico Tardío; sin embargo, no se ha confirmado la existencia de la capa de la TBJ en dicho sitio (Andrews, 1986: 33-76).

$\mathrm{Al}$ analizar los sitios arqueológicos con presencia de la capa de la TBJ primaria ubicados en el área del Sureste de la Caldera Ilopango, no se observa la reocupación inmediata después del evento volcánico de Ilopango entre el Siglo V y VI a excepción de Quelepa.

En este capítulo se revisaron respectivamente las estratigrafías antes y después de la caída de la TBJ primaria en los sitios arqueológicos en donde se ha registrado la presencia de la TBJ primaria.

Alrededor de la Caldera de Ilopango y el Valle de Las Hamacas habría ocurrido devastación total en cuanto al ambiente natural y el cultural.

En el área Este del Valle de Zapotitán, el área Norte de la región central de El Salvador y el área de Bajo Lempa y la Bahía de Jiquilisco, tampoco se observa la reocupación inmediata de los asentamientos humanos después de este evento natural catastrófico.

Por lo tanto, se puede establecer que la erupción del Caldera Ilopango que ocurrió entre el Siglo V y VI d.C. afectó severa y ampliamente en San Salvador y su contorno, el Valle de Zapotitán, el Norte de San Salvador, el área del Rio Jiboa, y los alrededores del Volcán San Vicente, así como el área fluvial del Río Lempa y la Bahía de Jiquilisco. 


\section{Tbj primaria registrada en la zona arqueológica chalchuapa}

La zona arqueológica Chalchuapa está localizada hacia el Oeste del Valle de Zapotitán, aproximadamente a $75 \mathrm{~km}$ al Noroeste de la Caldera Ilopango.

En dicha zona se llevaron a cabo ininterrumpidamente los estudios arqueológicos desde el año 1941 hasta la fecha. Especialmente después de 1995 se realizaron las excavaciones en las estructuras y sus alrededores en las áreas de Casa Blanca, Tazumal y El Trapiche, por lo cual se comprende la ubicación estratigráfica de la TBJ con relación a las arquitecturas prehispánicas.

En este capítulo se revisará cada punto donde se registró la capa de la TBJ dentro de la zona en mención y finalmente se interpretará qué es lo que estaba pasando antes y después de la erupción del Volcán Ilopango en la ciudad prehispánica de Chalchuapa.

La zona arqueológica Chalchuapa consiste en 10 áreas, las cuales son del Norte al Sur, Pampe, El Trapiche, Casa Blanca, Peñate, Las Victorias, Laguna Cuzcachapa, Tazumal, Nuevo Tazumal, Gavilán y Laguna Seca.

En las áreas de El Trapiche, Casa Blanca y Tazumal se ha registrado la presencia de la TBJ primaria (Sharer, 1978a: 71, Figs.8 Parte 1 y 50d; Sedat et.al., 1978: 105, Figs.26, 27 y 28; Ohi, 2000: 20, 27, 28, 33, 37 y 38; Shibata, 2005: 130, 139 y 147; Ito et.al., 2016: Figura I 11 (a) y (b), Foto. I 17 y 18). Además, en el fondo de la Laguna Seca y Laguna Cuzcachapa está registrada la sedimentación de la TBJ primaria (Hart et.al., 1983b: 42; Dull, 2004: 238) 


\section{Figura 5}

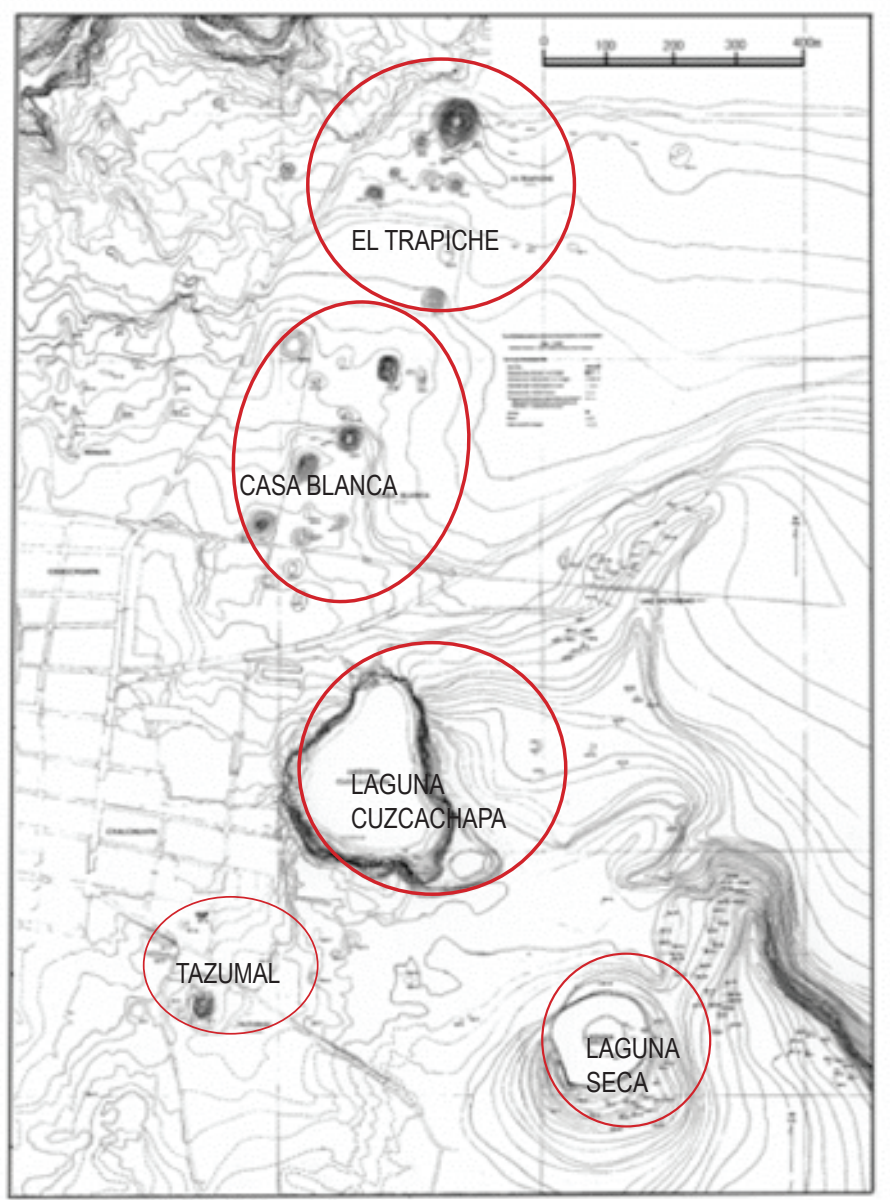

Ubicación de las áreas de El Trapiche, Casa Blanca, Tazumal, Laguna Seca y laguna Cuzcachapa en la zona arqueológica Chalchuapa.

En el área de El Trapiche la capa de TBJ primaria está localizada en la plaza rodeada por los Montículos E3-1, E3-2 y E3-3, la cual se depositó directamente sobre 3 monumentos de cabezas de jaguar, 2 fragmentos de estela y otras 2 esculturas de piedra (Sharer, 1978a: 67, 71 y Fig.8; Ito, 2014: 19-25 y Figura 26).

Una vasija en forma de cántaro de Guazapa engobe raspado se colocó como una ofrenda perforando la capa de la TBJ primaria (Sharer, 1978a: 71 y Fig.8 Parte 1; 1978b: 181). 
Sobre dicha capa se registró un piso de barro apisonado o argamasa en las Trincheras 1-3, 1-4 y 1-5 ubicadas en el área entre la Estructura E3-2, la E3-3 y la plaza al frente de la Estructura E3-1 (Ito et.al., 2014a: 14).

Por lo tanto, se puede indagar que la actividad humana tales como peregrinación ancestral en el área de El Trapiche continuó después de la caída de la TBJ primaria, aunque las estructuras de El Trapiche estaban abandonadas y no continuó el rito relacionado con las cabezas de jaguar estilizado en aquel entonces (Sharer, 1978a: 74 y Fig.8 Part 1; Ito et.al., 2014b: 28).

En el área de Casa Blanca se ha registrado la TBJ primaria en 7 lugares diferentes; al Este de la Estructura 5, al frente de la misma, al frente de la Estructura 1, al Norte de la Estructura 2, en la Trinchera 4N, en la Trinchera M1, en los pozos antes de construir el portón principal del parque.

En el corte estratigráfico mostrado en la Trinchera 4N, la cual es actualmente una ventana arqueológica, se observa una capa de la TBJ primaria, cuyo grosor oscila entre 16 y $18 \mathrm{~cm}$, sobre la cual se forman 3 capas de la TBJ secundaria. En total el grosor mide entre 40 y $50 \mathrm{~cm}$ (Kitamura, 2016a: 322; Kitamura, 2017: 308)

\section{Fotografia.4}

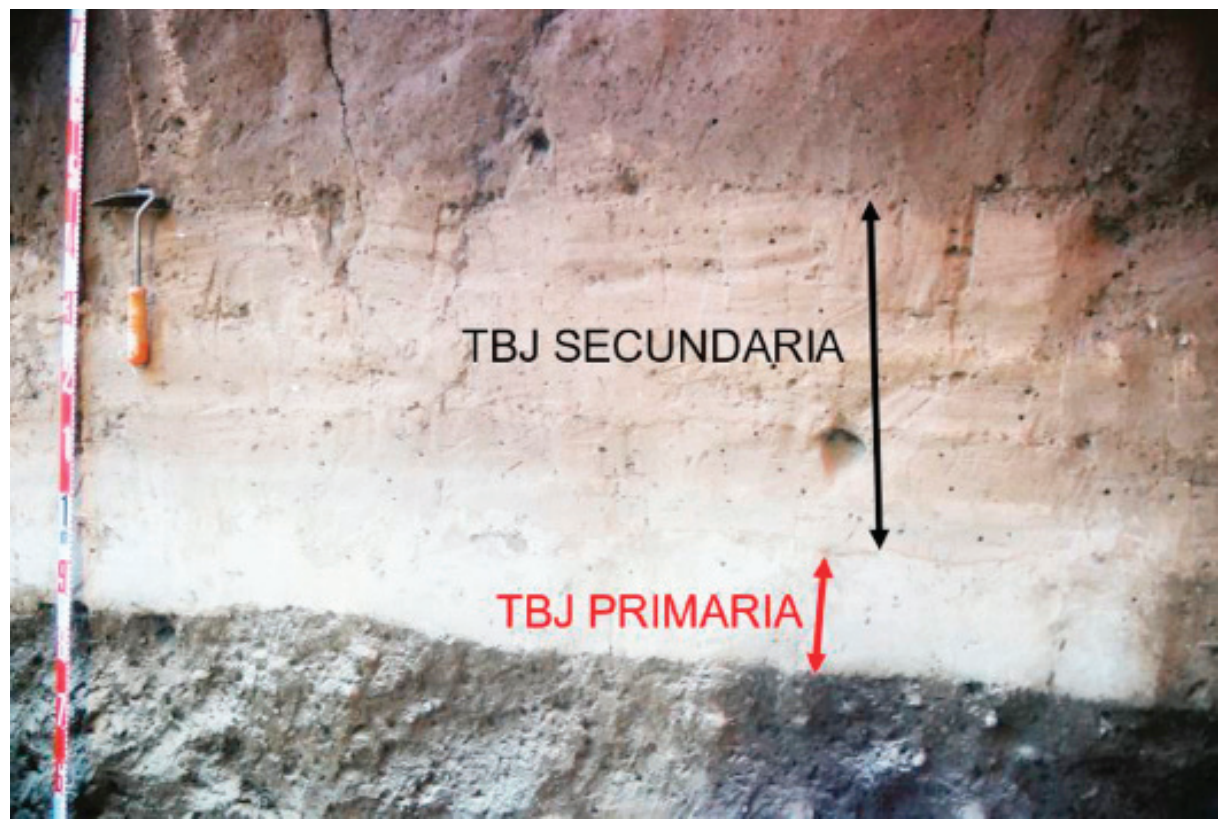

Capas de la TBJ primaria y secundaria observadas en la Trinchera 4N del Parque Arqueológico Casa Blanca. 
Los resultados de las excavaciones realizadas en el área de Casa Blanca desde el año 1995, indican que dicha área comenzó a habitarse durante el Período Preclásico Tardío como parte de la expansión urbanística que dio inicio desde el área de El Trapiche.

Posteriormente se construyó la gran-plataforma que mide $240 \mathrm{~m}$ de Norte a Sur y $220 \mathrm{~m}$ de Este a Oeste, sobre la cual se edificaron por lo menos la Subestructura 5 c y la Subestructura 2; sin embargo antes de la caída de la TBJ primaria el área de Casa Blanca fue abandonada. Durante el Período Clásico Temprano (300 600 años d.C.) el área de Casa Blanca dejó de funcionar como parte del centro ceremonial (Shibata et.al., 2002: 1008; Ito, 2010).

El área de Tazumal está ubicada en el sector más Sur de la zona arqueológica Chalchuapa. La construcción de estructuras en el área comenzó desde el final del Período Preclásico Tardío o el principio del Clásico Temprano (Shibata, 2005: 132 y 133; Ito et.al.2009: 20, Figura 44f y Foto 55)

\section{Fotografía 5}

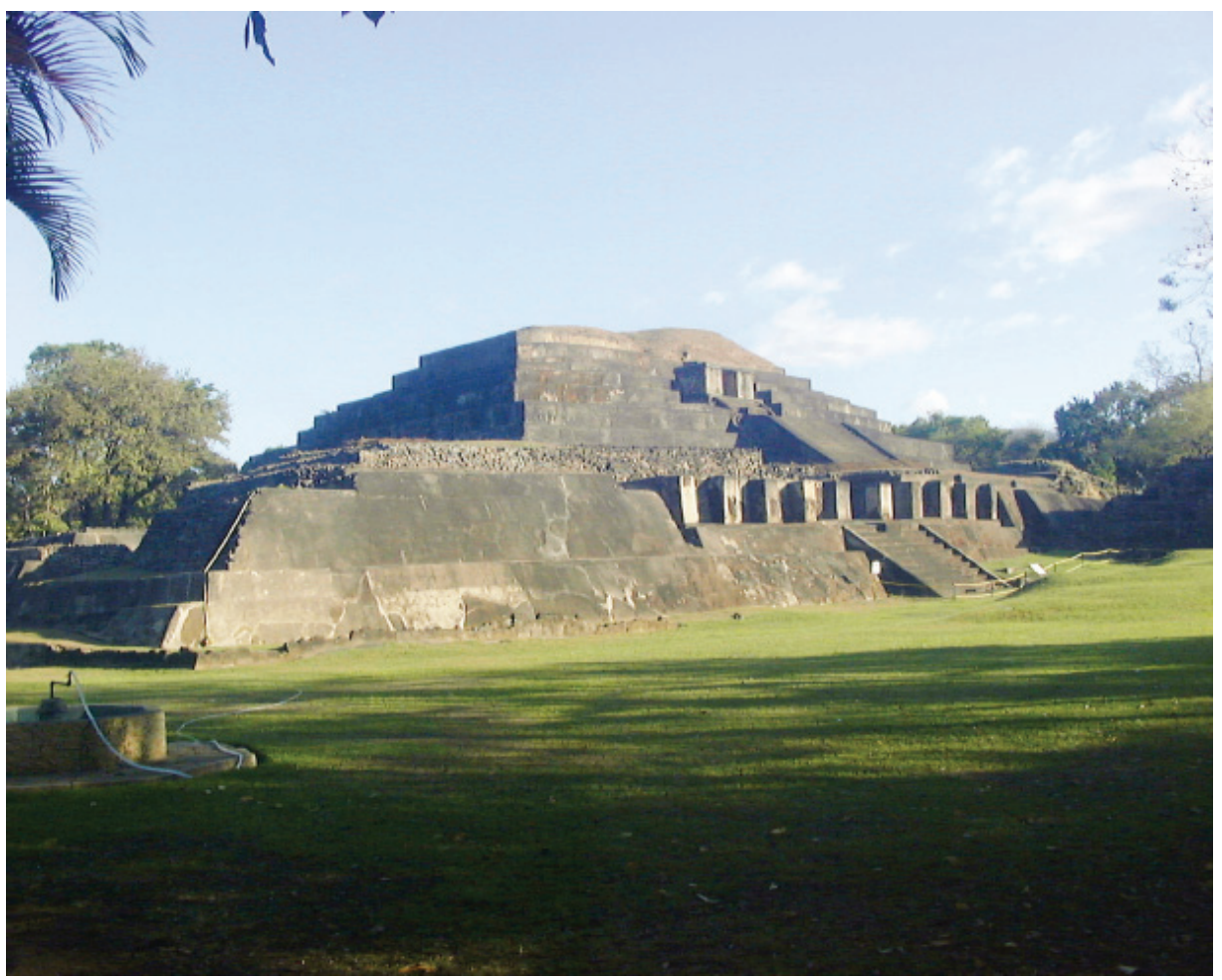

Complejo arquitectónico de Tazumal. 
En dicho sector se registró los restos de la TBJ primaria en 6 diferentes puntos dentro del complejo arquitectónico y sus alrededores

\section{Figura 6}

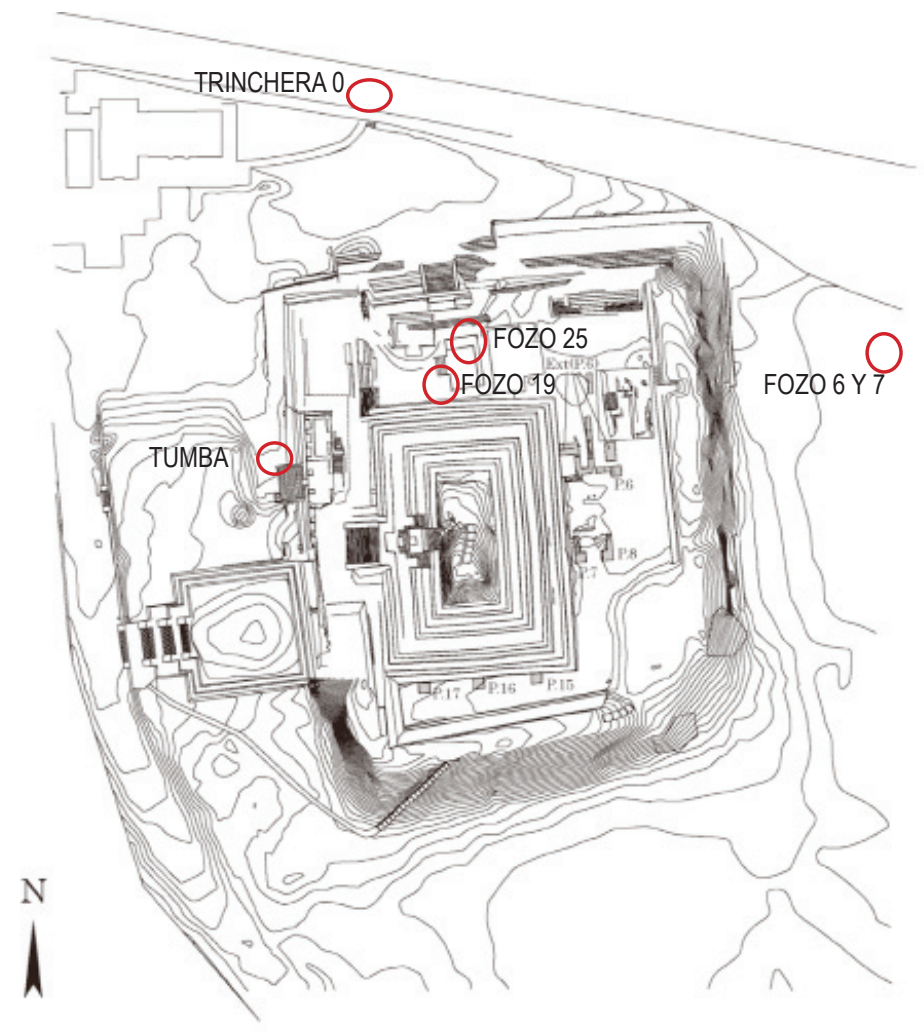

Figura 4 Pozos 6-8, 15-17 en el Parque Arqueológico de Tazumal

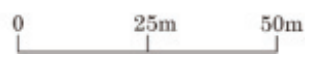

Ubicación de los 6 puntos en el área de Tazumal en donde está registrada la TBJ primaria.

En el Pozo 19 ubicado en la esquina Suroeste de la Estructura B1-1b (Foto.6) se registró la capa de la ceniza volcánica de color blanco, cuyo grosor mide aproximadamente $17 \mathrm{~cm}$ y se conserva la laminación estructural característica de la TBJ primaria (Shibata et.al., 2010: 832) 


\section{Figura 7}

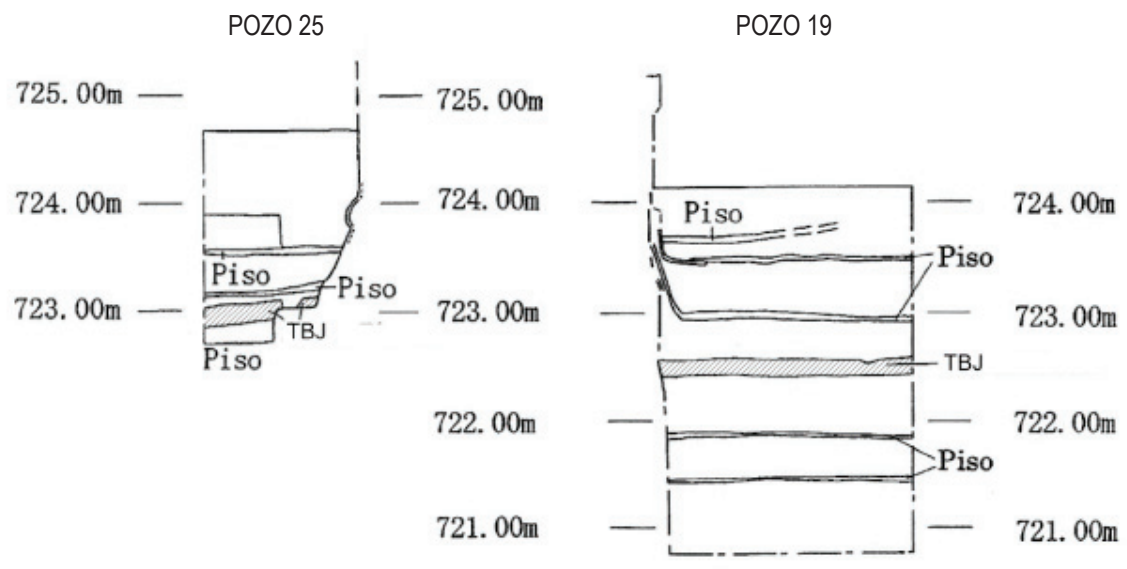

Cortes estratigráficos en el Pozo 19 y el 25 (Ito, 2009: Figura 13 modificada por el autor).

\section{Fotografia 7}

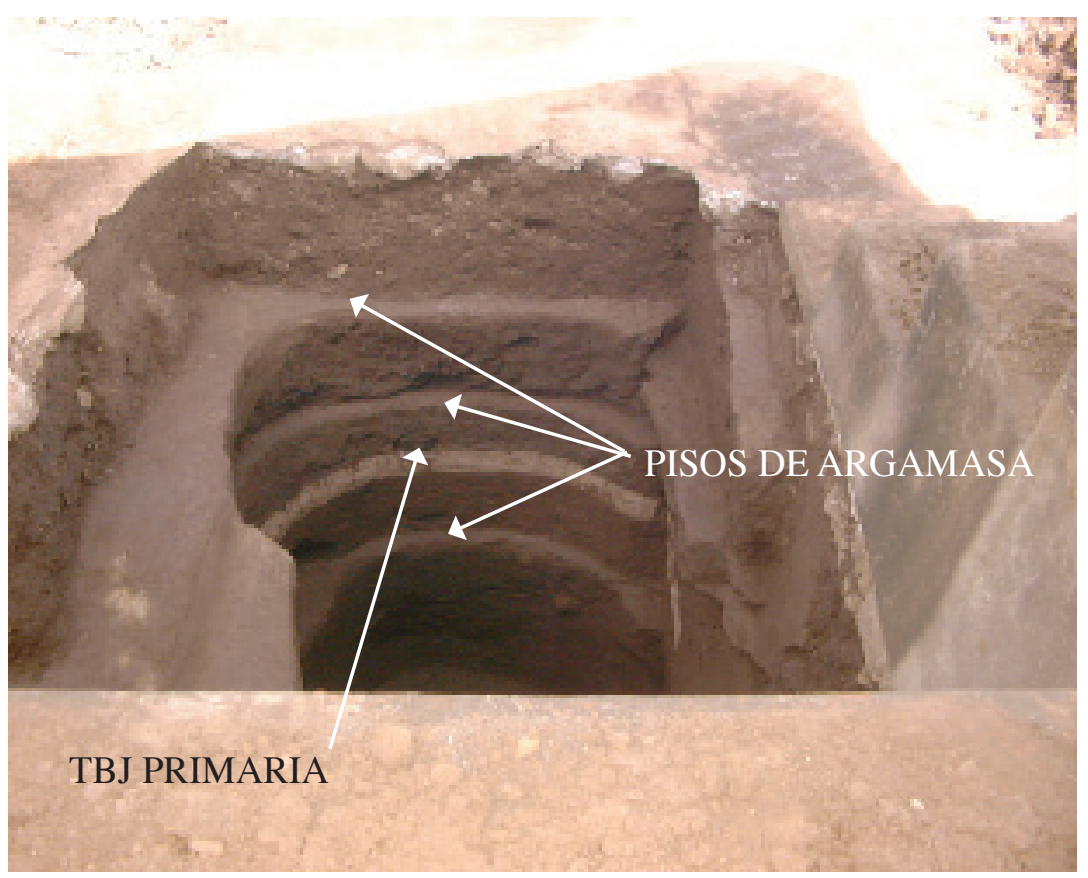

TBJ primaria encontrada en el Pozo 19 debajo de la Estructura B1-1b. 
Al Norte de la misma Estructura se excavó otro pozo denominado como Pozo 25, en donde se reconfirmó la capa de TBJ primaria (Kitamura, 2016a: 323; Shibata et.al., 2010: 832). Dicha capa mide aproximadamente $22 \mathrm{~cm}$ de grosor y consiste en 3 subcapas las cuales son de la inferior hacia la superior la B y $\mathrm{D}$, la E y la G, mostrando la estratigrafía muy característica de la TBJ primaria (Kitamura, 2016: 323 y Figura II. 16)

\section{Figura 6}

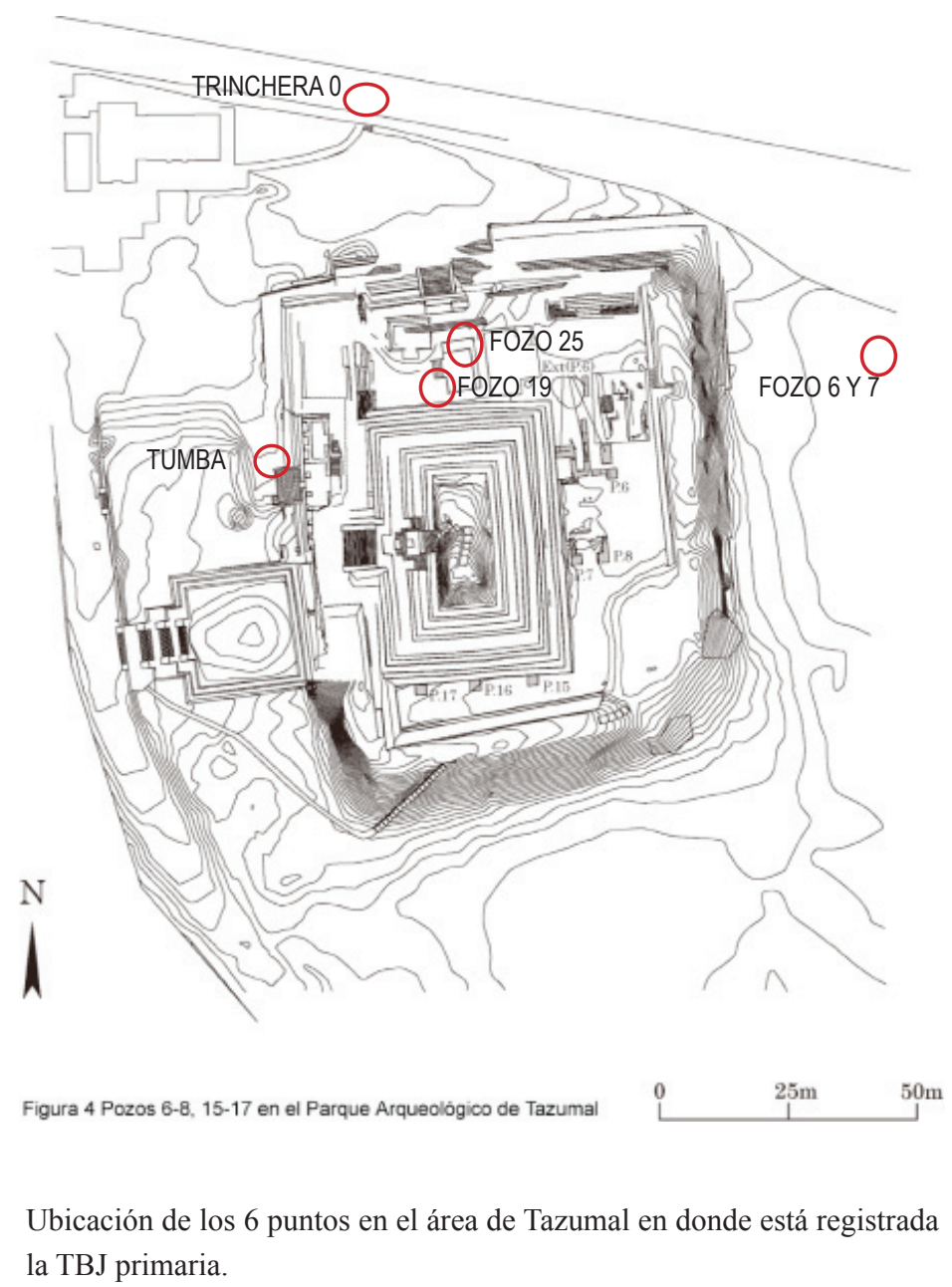

la TBJ primaria. 
Fotografía 8

\section{-RELLENO DE CONSTRUCCION}

\section{PISO DE ARGAMASA}

\section{RELLENO DE CONSTRUCCION}

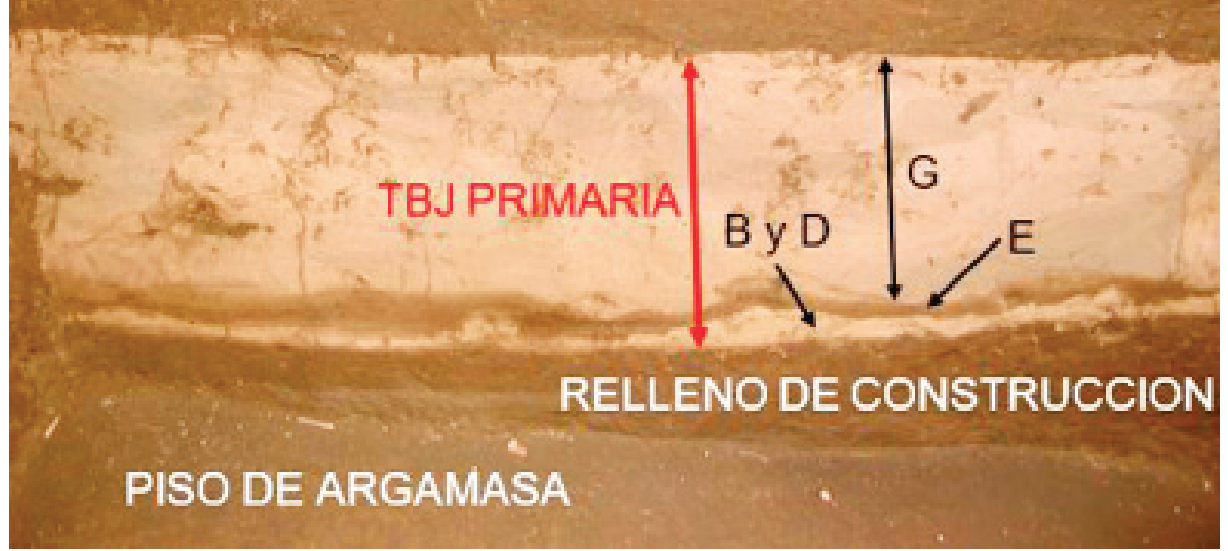

TBJ primaria encontrada en el Pozo 25.

Lo interesante es que la capa de la TBJ primaria registrada respectivamente en los Pozos 19 y 25 está intercalada entre las capas de la tierra del color café - negro, típico material de construcción para rellenar estructuras del Período Preclásico y Clásico en la zona arqueológica Chalchuapa. No se observa la capa de la tierra del color negro causada por el crecimiento de la vegetación sobre la TBJ primaria, lo cual implica que no habrían transcurrido varios años de abandono por lo menos en la Estructura B1-1b después de la erupción del Volcán Ilopango, sino que continuó la construcción en la Estructura de Talud con cornisa, imitando Talud - Tablero, el estilo arquitectónico característico de la cultura Teotihuacana.

En el dibujo del corte estratigráfico de la Tumba 13 perforada desde la banqueta de la Estructura B1-1d-III (Shibata et.al., 2008: 1067 y 1068), el cual está elaborado por Stanley H. Boggs, se indica la existencia de una capa de la ceniza volcánica del color blanco estéril debajo de la misma Estructura, cuyo grosor es de aproximadamente unos $40 \mathrm{~cm}$. 
Figura 8

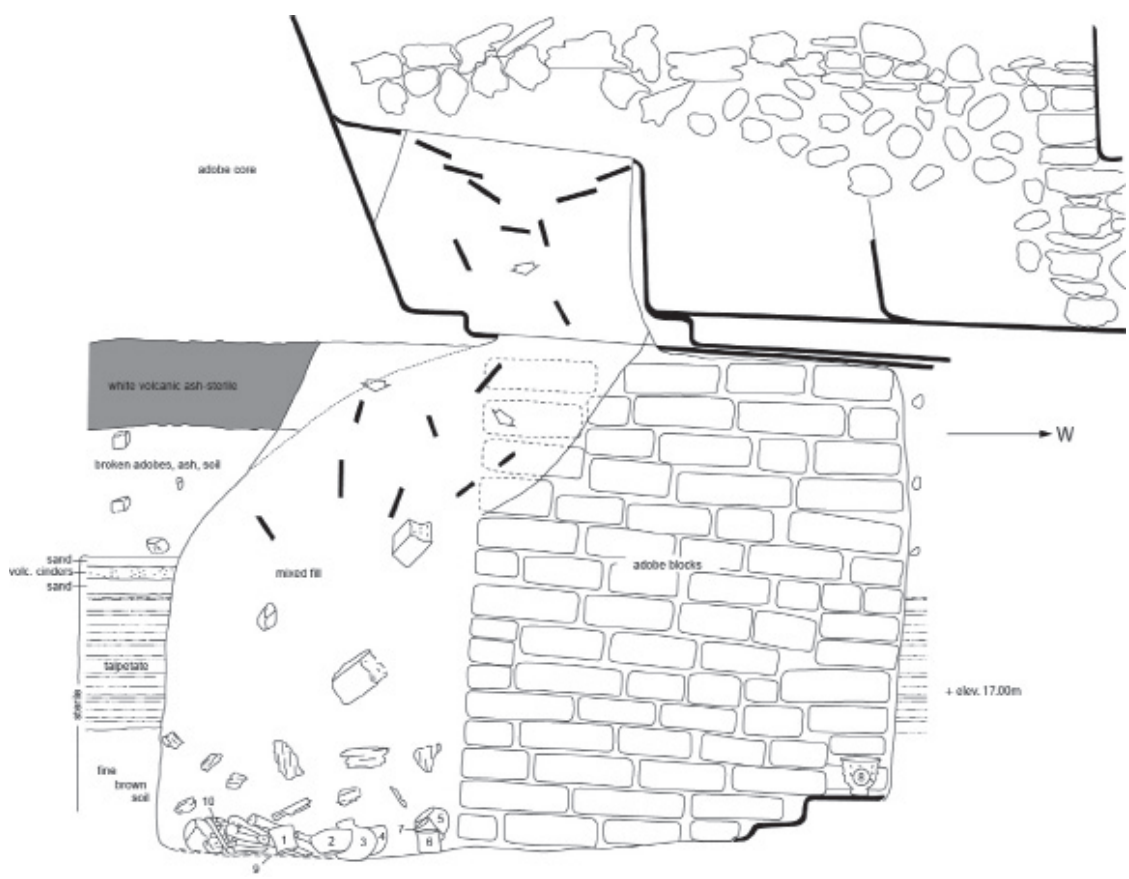

Corte estratigráfico de la Tumba 13 registrado por Stanley H. Boggs (calco digital elaborado por Akira Ichikawa).

El estudio de la TBJ en la zona arqueológica Chalchuapa presentado por Kitamura, Indica que el grosor de la TBJ primaria oscila entre 16 y $25 \mathrm{~cm}$ (Kitamura, 2007; Shibata et.al., 2010: 832; Kitamura, 2017: 43). Por lo tanto, la cifra $40 \mathrm{~cm}$ de grosor en el dibujo del corte estratigráfico arriba mencionado podría ser el grosor total de la TBJ primaria y la secundaria al igual que el caso observado en la Trinchera 4N del área de Casa Blanca.

Cabe mencionar que arriba de la capa de la ceniza volcánica blanca estéril en mención se indica solamente la capa del relleno de la Estructura (Fig.8).

La Trinchera 0 (Tr.0) ubicada al frente del busto monumental del Che Guevara fue una zanja excavada por la maquinaria de la alcaldía municipal de Chalchuapa con el fin de instalar tubos de desagüe para aguas lluvias a lo largo de la $7^{\mathrm{a}}$ Calle Oriente en el mes de diciembre del año 2000.

Inmediatamente se realizó un rescate arqueológico. Se excavaron 3 trincheras de 2 x 1 en planta aparte de la Tr.0 (Shibata, 2005). 
Resultó que se registraron 3 pisos de argamasa superpuestos, los cuales fueron denominados del inferior al superior Piso 3, Piso 2 y Piso 1.

El Piso 3 y 2 estaban en buen estado de conservación y el Piso 1 estaba más deteriorado. El Piso 2 fue observado en la distancia más larga comparando con los otros 2 pisos. En el medio de la Tr.0 se registró la capa de la ceniza volcánica del color blanco, la cual consiste en varias subcapas, cuyas inferiores presentaron las características típicas de la TBJ primaria. Las superiores podrían ser de la TBJ secundaria según la gradación del color desde el blanco hacia el amarillento (Ibid., 2005: 130, Fig.4 y Foto 8)

\section{Figura 9}

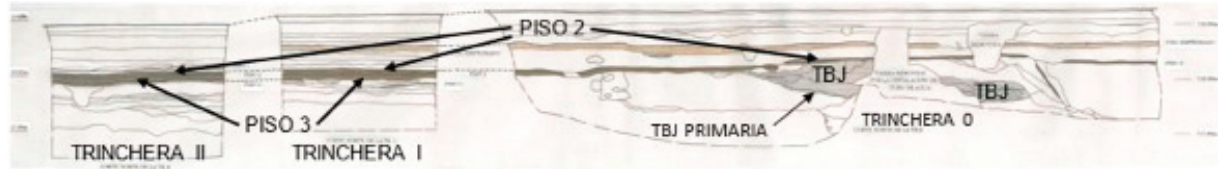

Cortes estratigráficos de la Trinchera 0 , Trinchera I y Trinchera II.

\section{Fotografía 9}

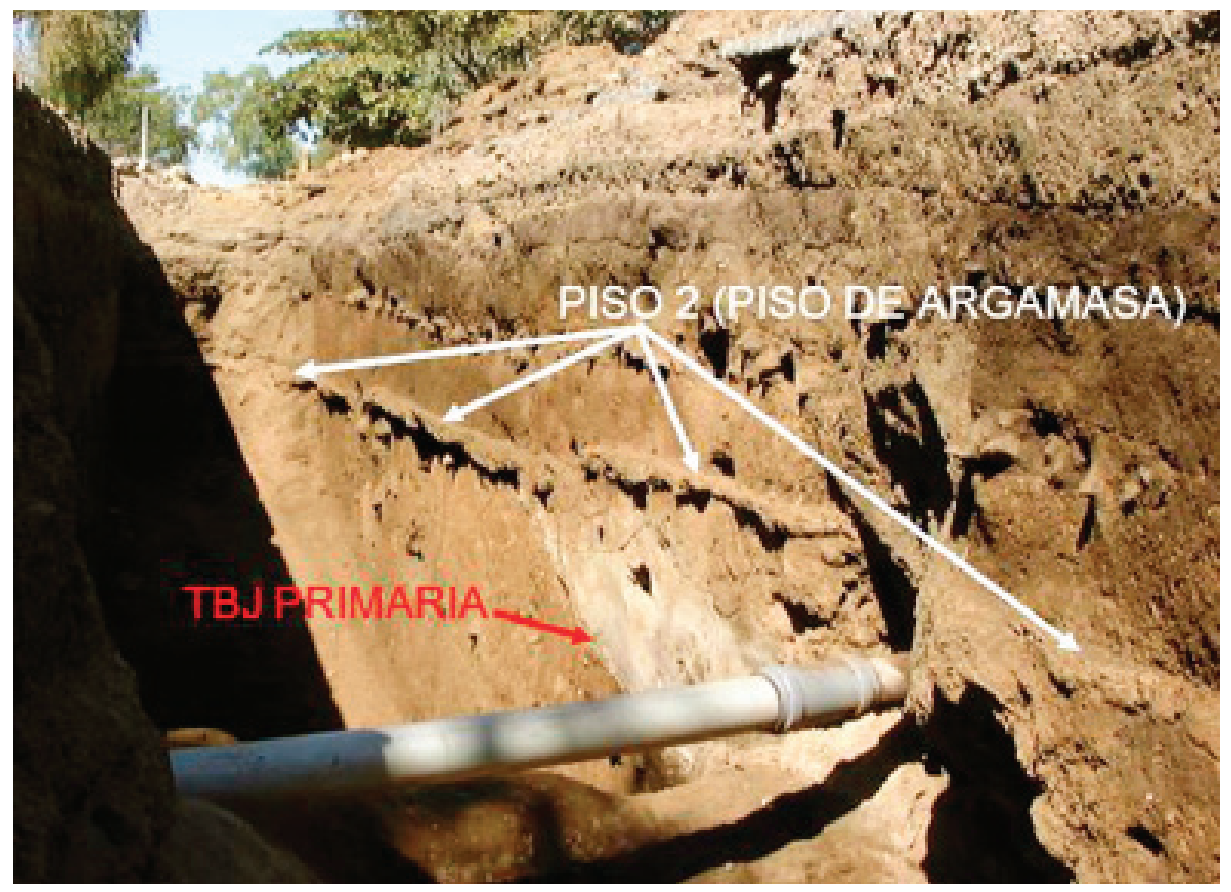

Capas de la TBJ primaria y secundaria observadas debajo del Piso 2 en la Trinchera 0. 
A consecuencia del rescate arqueológico realizada en la $7^{\text {a }}$ Calle Oriente al Norte del Parque Arqueológico Tazumal, se podría interpretar que después de construir el Piso 3 habría caído la ceniza volcánica de Ilopango, no obstante, se hizo la ampliación del piso de argamasa (Piso 2), ya que no se observa capa de la tierra del color negro entre el Piso 3 y 2, lo cual podría implicar el abandono del área de Tazumal durante muchos años (Fig.9; Foto.9).

En el año 2016 se realizaron pozos de sondeo en el sector al Este de la Gran Pirámide B1-1 antes de la construcción de servicio sanitario con ducha para los empleados del Parque Arqueológico Tazumal.

Durante las excavaciones se registró la capa de la TBJ primaria con grosor a unos $20 \mathrm{~cm}$ en los Pozos 6 y 7, la cual fue cubierta por otra capa del color blanco-beige aproximadamente a $1 \mathrm{~m}$ de grosor sin interrupción de otra capa intercalada (Foto. 10 y 11).

\section{Fotografia 10}

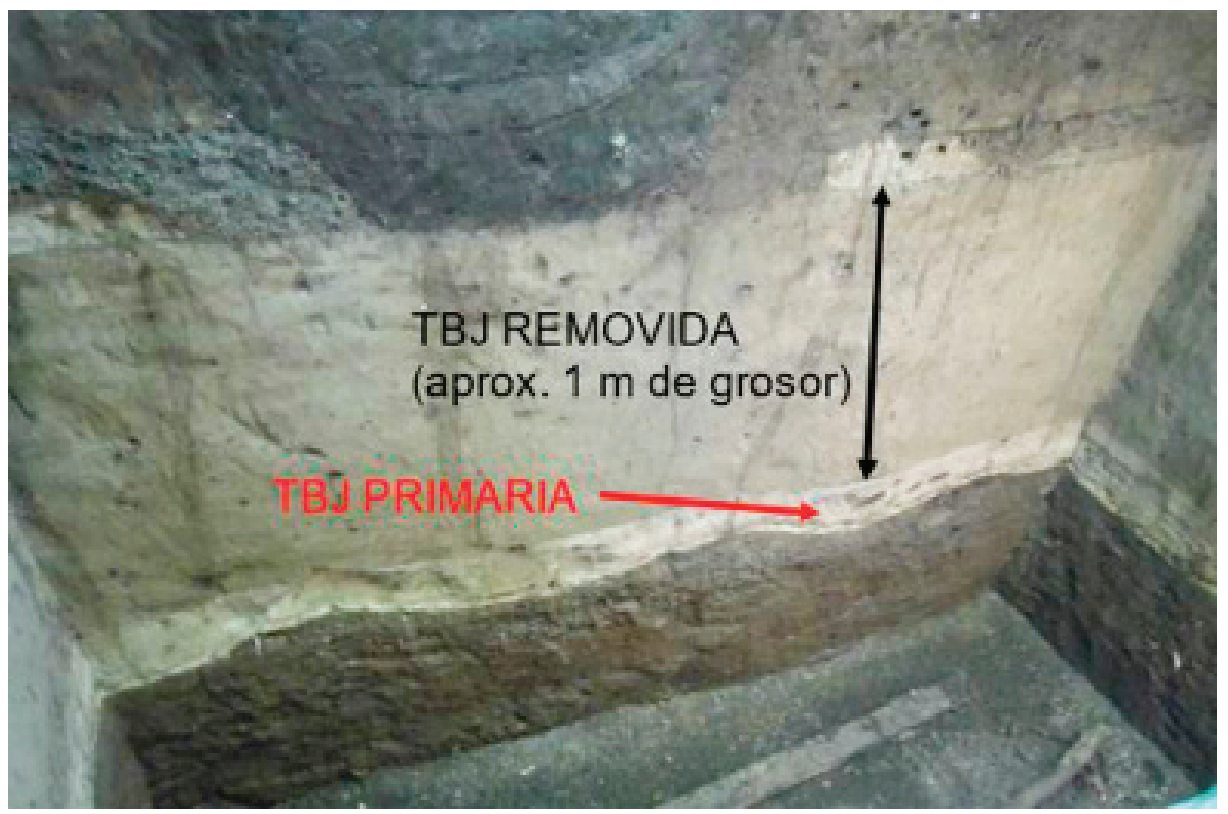

Corte estratigráfico del Pozo 6 


\section{Fotografia 11}

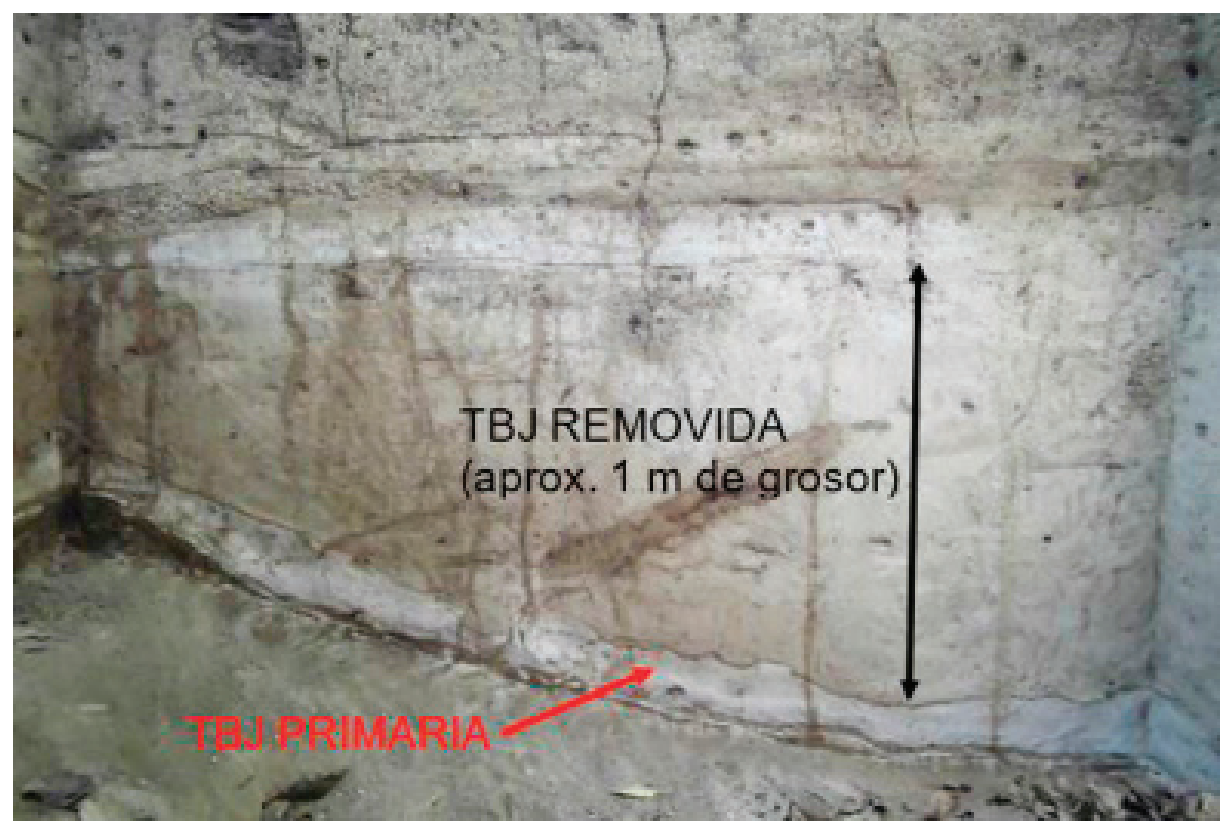

Corte estratigráfico del Pozo 7

Según comentarios de dos vulcanólogos, uno Español y otro Japonés, quienes observaron dichas estratigrafías, las gruesas capas en mención podrían ser de la TBJ removida por causa antrópica (Comunicación personal con Iva Suñe Puchol y Shigeru Kitamura).

En este capítulo se mostraron las huellas de la TBJ primaria registradas en la zona arqueológica Chalchuapa, la mayoría de las cuales presentan relación estratigráfica con el contexto arquitectónico.

Al comparar con las áreas devastadas por la erupción del Caldera Ilopango entre el Siglo V y VI d.C. tales como San Salvador y su contorno, Valle de Zapotitán, Norte de San Salvador, el área del Rio Jiboa, alrededor del Volcán San Vicente, el área fluvial del Río Lempa y la Bahía de Jiquilisco, el caso de la zona arqueológica Chalchuapa demuestra un contraste destacado.

En el área de Tazumal continuó la construcción del complejo arquitectónico y sus alrededores, haciendo la remoción de la TBJ acumulada sobre la ciudad prehispánica y posteriormente edificando el templo principal más grande que los anteriores. 


\section{Conclusión}

Los puntos principales de discusión que se tratan en este artículo son dos: uno es la influencia ante la naturaleza y la sociedad, provocada por la erupción del Volcán Ilopango entre el Siglo V y VI d.C. y el otro es el fechamiento absoluto de dicho evento volcánico.

La mayoría de los estudios hasta la fecha sobre el evento volcánico de la caldera Ilopango ocurrido entre el Siglo V y VI, están basados principalmente en las ciencias naturales tales como la geología y la vulcanología. No se ha tomado en cuenta la observación y análisis de los estratos debajo y arriba de la capa de la TBJ primaria registrados en los sitios arqueológicos a través de las investigaciones realizadas.

Como consecuencia de los estudios desde el punto de vista arqueológico, la región que abarca San Salvador y su contorno, Valle de Zapotitán, Norte de San Salvador, el área del Río Jiboa, alrededor del Volcán San Vicente, el área fluvial del Río Lempa y la Bahía de Jiquilisco, estaba devastada por la erupción de la caldera Ilopango. La reocupación de los sitios podría depender del impacto físico causado por el evento volcánico y esperaría hasta el principio del Período Clásico Tardío.

Al contrario, en la zona arqueológica Chalchuapa, especialmente en el área de Tazumal no se observó ninguna señal de abandono durante largo tiempo más de unos años.

El complejo del templo principal estaba en proceso de la nueva construcción al momento de la caída de la TBJ; sin embargo, dichas labores de edificación no fueron suspendidas por un lapso de tiempo largo. Posiblemente después de que se depositó la ceniza volcánica de la TBJ en la ciudad prehispánica de Chalchuapa, los habitantes comenzarían la remoción de dicha capa (Foto.10 y 11) bajo la dirección del dirigente de la sociedad, la cual podría ser muy organizada más de lo que se piensa actualmente.

La construcción del complejo del templo principal en Tazumal reinició (Shibata et.al., 2010: 832)(Fig.9), al frente del cual se habría ejecutado la pavimentación de argamasa, ampliándola más que el tiempo antes de la erupción del Volcán Ilopango (Shibata, 2005: 130, Fig.4 y Foto. 8).

Posteriormente se desarrolló la edificación de los templos principales cada vez más grandes, cubriéndolos respectivamente mediante la construcción posterior (Shibata et.al., 2008: 1068) y el área del centro ceremonial se extendería 
más hacia el área de Casa Blanca hasta el final del Período Clásico Tardío, aproximadamente a 950 años d.C.

Por otro lado, el fechamiento absoluto del evento natural en mención se ha expuesto con base en el análisis de C14.

A mediados de los 1970's y principios de los 1980's Sheets presentó la datación de la erupción del Volcán Ilopango como 260 años \pm 114 d.C. mediante análisis de C14 (Sheets 1983: 5).

Dicha datación se establece al final del Período Preclásico Tardío o el Período Protoclásico, por lo tanto, diversos arqueólogos de aquel entonces interpretaron que el evento volcánico de la Caldera Ilopango causó decadencia de la Región Sur Maya y la migración hacia las Tierras Bajas Mayas y podría ser el marcador de la transición desde el Período Preclásico o el Protoclásico al Clásico Temprano (Sheets, 176: 59 y 61; Sharer, 1978c: 210 y 211; Lowe, 1982: 28; Parsons 1986: 97).

A inicios del Siglo XXI Dull y sus investigadores presentaron otro fechamiento absoluto de la erupción del Volcán Ilopango utilizando AMS, una técnica más avanzada de datación. El fechamiento se encuentra dentro del rango entre 408 (429) 536 años d.C. (Dull et.al. 2001:28).

Posteriormente Dull realizó los estudios estratigráficos de los sedimentos en las 4 lagunas en la cuenca del Río Paz y la Sierra de Apaneca, ubicadas en el Occidente de El Salvador con el objetivo de conocer el ambiente natural antes y después de la erupción del Volcán Ilopango. Concluyó que el fechamiento absoluto de dicho evento volcánico podría ser 430 años d.C (Dull, 2004: 238).

En el año 2010 Dull y sus colegas presentaron otro fechamiento absoluto de la erupción del Volcán Ilopango, el cual fue basado en el análisis de las 18 nuevas muestras de carbón (Dull et.al., 2010).

Al mismo tiempo el nuevo fechamiento se relaciona con el evento de carga del aerosol atmosférico más grande ocurrido en ca. 536 años d.C. Dicho evento fue una niebla seca que envolvió gran parte de la esfera terrestre y provocó un enfriamiento global prolongado, el cual había durado desde ca. 536 años d.C. hasta ca. 550 años d.C.

Según registros de anillos de crecimiento de árboles, cuentas históricas y datos arqueológicos, los efectos fueron aparecidos en el Hemisferio Norte. Con base en una revisión sobre las cronologías del núcleo de hielo y los registros de deposición de sulfato volcánico $\left(\mathrm{SO}_{4}\right)$ recolectados en las áreas de la 
Antártida (LMD) y Groenlandia (Dye-3, GRIP y NGRIP), Dull y los científicos concluyeron una gran erupción volcánica en la región tropical del Hemisferio Norte como fuente probable, la cual podría ser la erupción del Volcán Ilopango (ibid., 2010).

Pero en caso de que se acepte el fechamiento de 536 años d.C. para la erupción de la Caldera Ilopango, sucedería un problema de concordancia con los tiempos de duración desde la Estructura B1-1d-III denominada como Edificio de las Columnas hasta la B1-1d-VII en el área de Tazumal (Shibata et.al., 2008: 1065, 1067-1069).

El inicio de la construcción del Edificio de las Columnas podría corresponder a 536 años d.C., ya que la estructura B1-1d-III está edificada sobre la capa de la TBJ.

Por otro lado, después de dejar la función de la Estructura B1-1d-VII y antes de la construcción de la subestructura de la Gran Pirámide B1-1 (ibid., 2008: 1068), se colocó en la esquina Sureste de la misma una ofrenda de un vaso cilíndrico con un cuenco polícromo como tapadera dentro del cual se pusieron 2 plaquetas de jadeíta, 50 fragmentos pequeños de jadeíta, huesos de aves, fragmentos de moluscos, mica y pigmento rojo (Ito et.al., 2007: 718).

La datación de dicha actividad ritual es entre ca. 547 y 632 años d.C. (2 sigmas) basado en el análisis de C14 utilizando una muestra de carbón recolectada debajo de la ofrenda arriba mencionada (Ichikawa, 2016: 344).

Si se toma en cuenta el fechamiento medio entre ca. 547 y 632 años d.C. (589 años d.C.) cuando se colocó la ofrenda arriba mencionada, durante unos 50 años se habría ejecutado 5 veces la remodelación del complejo del templo principal en Tazumal, lo cual significa que se habría construido un complejo del templo principal aproximadamente cada 10 años.

Al comparar este caso con diversas construcciones de templos piramidales en Mesoamérica, el lapso del tiempo cuando un templo principal esté en función sería demasiado corto.

Además, los complejos del templo principal en Tazumal posteriores de la caída de la TBJ serían cada vez más grandes. Es decir que no podría observarse el decaimiento de la sociedad de Tazumal después de la erupción del Volcán Ilopango. 
El Dr. Hirotaka Oda del entonces Centro de Investigación de Datación de la Universidad de Nagoya realizó un fechamiento de la TBJ a través del análisis de C14 con la muestra de la parte externa del tronco procedente del sitio arqueológico El Mico, municipio de Guazapa, departamento de San Salvador y en el año 2013 presentó el resultado de fechamiento como ca. 419 (436, $489,511,516,530) 544$ años d.C. Esta datación se muestra la semejanza al fechamiento del rango entre ca. 408 (429) 536 años d.C. presentado por Dull y sus colegas en 2001.

Al considerar los 2 puntos arriba mencionados, el fechamiento absoluto de la erupción del Volcán Ilopango que afectó a los habitantes de la Época Prehispánica, podría ser la primera mitad del Siglo V d.C.

Cabe mencionar que durante el lapso de tiempo entre 430 y 530 años d.C. la curva calibrada de C14 oscila casi horizontalmente, por lo tanto, aunque sea más precisa la datación de $\mathrm{C} 14$ a través de AMS, el fechamiento calibrado presentará más diferencia, por ejemplo, con unos 10 años de diferencia desde el presente, el fechamiento calibrado mostrará casi 100 años de margen de diferencia (Shibata et.al. 2010: 838, Figura 3).

Es necesario buscar otro método físico-químico para la datación. En este punto de vista el Vulcanólogo Shigeru Kitamura ya coordinó otro tipo de fechamiento, el cual está en proceso.

\section{Agradecimiento}

Para elaborar este artículo nos apoyaron el Dr. Nobuyuki Ito, Dr. Shigeru Kitamura, Dr. Akira Ichikawa a través de las investigaciones realizadas por ellos. La Arqlga. Michelle Toledo arregló las figuras y fotos utilizadas en este artículo. El Arqlgo. Oscar Camacho y Arqlga. Margarita Morán nos hicieron favor de chequear la descripción del idioma español. El Dr. Ramón Rivas nos dio la gran oportunidad de publicar este artículo en la Revista de Museología Kóot del Museo Universitario de Antropología de la Universidad Tecnológica de El Salvador. Aquí les expresamos nuestro profundo agradecimiento a mis compañeros. Muchas gracias. 


\section{Referentes bibliográficos}

Amaroli, Paul y Robert Dull A.(1999). Milpas prehispánicas en El Salvador. En XII Simposio de Investigaciones Arqueológicas en Guatemala, 1998 (editado por J.P. Laporte y H.L. Escobedo), pp.562-572. Museo Nacional de Arqueología y Etnología, Guatemala.

Andrews V., E. Wyllys, (1986). La arqueología de Quelepa. Dirección de Publicaciones e Impresos Ministerio

de Cultura y Comunicaciones. San Salvador, El Salvador, C.A.

Begley, Christopher, Timothy Sullivan, Linda Brown, Aixa Wilson \& Kathryn Sampeck, 1996. Proyecto Arqueológico San Andrés Tomo I (Informe entregado ante la Dirección Nacional de Patrimonio Cultural de la CONCULTURA). Patronato Pro- Patrimonio Cultural, San Salvador, El Salvador C.A.

Begley, Christopher, Roberto Gallardo, Jeb Card, Aixa Wilson, Linda Brown \& Nicholas Herrmann, (1997). Proyecto Arqueológico San Andrés (Informe entregado ante la Dirección Nacional de Patrimonio Cultural de la CONCULTURA). Patronato Pro- Patrimonio Cultural, San Salvador, El Salvador C.A.

Boggs, Stanley H. (1943). Notas sobre las excavaciones en la Hacienda "San Andrés, Departamento de La Libertad". En TZUNPAME octubre de 1943 Año III, Número I, pp.104 - 126, Museo Nacional de El Salvador, El Salvador C.A. 1966. "Pottery Jars from the Loma de Tacuazin, El Salvador". Preprinted from Publication 28 : pp.175-185, Middle American Research Institute, Tulane University, New Orleans, E.U.A.

Dull, Robert A. (2004). Lessons from the mud, lessons from the Maya: Paleoecological records of the Tierra Blanca Joven eruption. En Geological Society of America, Special Paper 375, pp. 237-244. E.U.A.

Dull, Robert A., John R. Southon \& Payson D. Sheets, (2001). "Volcanism, Ecology and Culture: a Reassessment of the Volcan Ilopango TBJ. Eruption in the Southern Maya Realm". En Latin American Antiquity, 12(1), pp. 25-44. Society for American Archaeology. E.U.A.

Dull, Roberto A., John Southon, Steffen Kutterolf, Armin Freundt, David Wahl \& Payson Sheets. (2010). "Did the Ilopango TBJ eruption cause the AD 536 Event?". En American Geophysical Union Fall Meeting abstract 2010. San Francisco. E.U.A.

Earnest Jr., Howard H. (1976). "Investigaciones efectuadas por el Proyecto No.1, Programa de Rescate Arqueológico Cerrón Grande, en la Hacienda Santa Barbara, Depto. de Chalatenango". En ANALES del Museo Nacional de Antropología "David J. Guzmán No.49, pp.57-73. Museo Nacional "David J. Guzmán”.Ministerio de Educación. El Salvador C.A. Erquicia Cruz, José Heriberto, Walter Hernández, Shintaro Suzuki \& Akira Ichikawa. (2016). "Observaciones del entierro documentado en Cerro 
El Carmen, San Pedro Perulapán, El Salvador”. En XXIX Simposio de Investigaciones Arqueológicas en Guatemala 2015 (editado por B. Arroyo L. M. Salinas y G. Ajú Álvarez), Tomo I, pp.561-566. Museo Nacional de Arqueología y Etnología. C.D. de Guatemala. Guatemala.

Hart, William J. E. \& Virginia Steen-McIntyre (1983a) "2. Tierra Blanca Joven Tephra from the AD 260 Eruption of Ilopango Caldera". En P.D. Sheets (Ed.) Archaeology and Volcanism in Central America, the Zapotitán Valley of El Salvador, pp. 14-34. University of Texas Press. Austin.E.U.A. Hart, William J. E. y Virginia Steen-McIntyre 1983b. "Appendix 2-A. Field Data for Geologic Stops Mentioned in Chapter 22". En P.D. Sheets (Ed.) Archaeology and Volcanism in Central America, the Zapotitán Valley of El Salvador, pp. 35-43. University of Texas Press. Austin. E.U.A.

Hernández, E. Walter, (2004). Características geométricas y vulcanológicas de las tefras de Tierra Blanca Joven, Caldera de Ilopango, El Salvador. Tesis de Maestría en Tecnológicas y Geológicas Universidad Politécnica de Madrid.

Herodier, Gustavo.(1997). San Salvador: el esplendor de una ciudad 18801930. Trade Litho, Inc., Florida, E.U.A.

Ichikawa, Akira. (2011). Estudio arqueológico de Nueva Esperanza, Bajo Lempa, Usulután. Colección Arqueología No.2. Dirección de Publicacones e Impresos, Secretaría de Cultura de la Presidencia. San Salvador, El Salvador C.A.

Ichikawa, Akira. (2015). "Antes de la erupción del Volcán Ilopango en el Bajo Lempa, El Salvador". En XXVIII Simposio de Investigaciones Arqueológicas en Guatemala 2014. (editado por B. Arroyo L. M. Salinas y L. Paiz), Tomo I, pp.423-432. Museo Nacional de Arqueología y Etnología. C.D. de Guatemala. Guatemala.

Ichikawa, Akira. (2016). "Apéndice 1: Fechamiento". En Informe Final de las Investigaciones Arqueológicas en el área de Tazumal de la zona arqueológica de Chalchuapa (editado por N. Ito: Informe entregado al Departamento de Arqueología de la Secretaría de Cultura de la Presidencia), pp.344-349. San Salvador, El Salvador, C.A.

Ichikawa, Akira \& Oscar Camacho, (2016). "Investigaciones recientes en el sitio arqueológico San Andrés, El Salvador". En XXIX Simposio de Investigaciones Arqueológicas en Guatemala 2015. (editado por B. Arroyo, L. M. Salinas y G. Ajú Álvarez), Tomo I, pp.551-559. Museo Nacional de Arqueología y Etnología. C.D. de Guatemala. Guatemala.

Ito, Nobuyuki (2009) Informe final de las investigaciones arqueológicas en Tazumal, 2004 - 2008, (Informe entregado al Departamento de Arqueología de la SECULTURA). San Salvador, El Salvador C.A.

Ito, Nobuyuki (2010). Casa Blanca, Chalchuapa (2000-2003), Universidad Tecnológica de El Salvador. San Salvador, El Salvador, C.A. 
Ito, Nobuyuki (2014). "5. Esculturas encontradas en El Trapiche". En Informe final del Proyecto "Investigación arqueológica a través de sondeo geofísico en el área de El Trapiche, Chalchuapa (2012 - 2014), pp.19-25, (Informe entregado al Departamento de Arqueología de la SECULTURA. San Salvador El Salvador, C.A.

Ito, Nobuyuki (2016). Informe Final de las Investigaciones Arqueológicas en el área de Tazumal de la zona arqueológica de Chalchuapa (Informe entregado al Departamento de Arqueología de la Secretaría de Cultura de la Presidencia). San Salvador, El Salvador, C.A.

Ito, Nobuyuki y Shione Shibata. (2007). "Las investigaciones arqueológicas en la Estructura B1-1, Tazumal, 2005-2006". En XX Simposio de Investigaciones Arqueológicas en Guatemala, 2006 (editado por J. P. Laporte, B. Arroyo y H. E. Mejia), Tomo II, pp.709-722. Museo Nacional de Arqueología y Etnología. C.D. de Guatemala. Guatemala.

Ito, Nobuyuki y Shione Shibata. (2009) "II. Investigaciones". En Informe final de las investigaciones arqueológicas en Tazumal, 2004 - 2008 (Informe entregado al Departamento de Arqueología de la SECULTURA), pp.1020. San Salvador, El Salvador C.A.

Ito, Nobuyuki y Shione Shibata. (2014a) "4. Excavaciones en El Trapiche". En Informe final del Proyecto "Investigación arqueológica a través de sondeo geofisico en el área de El Trapiche, Chalchuapa (2012 - 2014), pp.12-18, (Informe entregado al Departamento de Arqueología de la SECULTURA. San Salvador El Salvador, C.A.

Ito, Nobuyuki y Shione Shibata. (2014b). "7. Conclusión”. En Informe final del Proyecto "Investigación arqueológica a través de sondeo geofísico en el área de El Trapiche, Chalchuapa (2012 - 2014), pp.28 y 29, (Informe entregado al Departamento de Arqueología de la SECULTURA. San Salvador El Salvador, C.A.

Ito, Nobuyuki y Shione Shibata. (2016) "I.3. EXCAVACIONES". En Informe Final de las Investigaciones Arqueológicas en el área de Tazumal de la zona arqueológica de Chalchuapa (Informe entregado al Departamento de Arqueología de la Secretaría de Cultura de la Presidencia), pp.15-103. San Salvador, El Salvador, C.A.

Kitamura, Shigeru. 2001. "IV: Tephra studies around the Casa Blanca archaeological site". En N. Ito (Ed.). Informe de la Primera y Segunda Temporada en el área de Casa Blanca del sitio Arqueológico Chalchuapa, El Salvador", pp. 52-56). (Informe preliminar entregado a la Unidad de Arqueología del Consejo Nacional para la Cultura y el Arte, CONCULTURA. San Salvador. El Salvador, C.A.

Kitamura, Shigeru. (2007). "Reevaluation of the Influence of a Gigantic Eruption from the Ilopango Caldera to Ancient mesoamerica Societies". Presentación de póster en Cities on Volcano 5 Conference Shimabara, Japón. 2010 “III. Estudios de la geomorfología volcánica y la estratigrafía 
de tefra alrededor de la Ciudad de Chalchuapa”. En N. Ito (Ed.), Casa Blanca, Chalchuapa (2000- 2003), pp. 249-256. Universidad Tecnológica de El Salvador. San Salvador. El Salvador, C.A.

Kitamura, Shigeru. (2016a) "II. Estudio Vulcanológico". En el Informe Final de las Investigaciones Arqueológicas en el área de Tazumal de la zona arqueológica de Chalchuapa (ed. N. Ito), pp. 300-343. (Informe entregado al Departamento de Arqueología de la Secretaría de Cultura de la Presidencia). San Salvador, El Salvador, C.A.

Kitamura, Shigeru. (2016b) "Influence of Ilopango Caldera eruption to the formation of San Juan del Gozo, Barrier, El Salvador, C.A." En MicroLandforms: The key to Connect Humans and Nature) (editado por K. Fujimoto, T. Miyagi, K. Saijo y Y. Takeuchi), pp.251-266. Kokon-Shoin Publisher, Tokyo, Japón.

Kitamura, Shigeru. (2017) "III. ESTUDIO VULCANOLÓGICO”. En el Informe Preliminar de Primera, Segunda, Tercera y Cuarta Temporada, Proyecto Arqueológico de El Trapiche, Chalchuapa (ed. N. Ito), pp. 3950. (Informe entregado al Departamento de Arqueología de la Secretaría de Cultura de la Presidencia). San Salvador, El Salvador, C.A.

Kitamura, Shigeru, Hirotaka Oda \& Naoto Yamamoto. (2007). "Paleoforest buried by a pyroclastic flow from the gigantic eruption during the third to fifth centuries, El Salvador Central America”. En Japanese Journal of Historical Botany Vol.15 No.1, pp.55-57. Japanese Association of Historical Botany, Tokyo, Japón.

Lardé, Jorge.(1950). "Cronología Arqueológica de El Salvador”. En ANALES, Tomo I No. 3, pp.72-79. Museo Nacional "David J. Guzmán”, San Salvador, El Salvador, C.A.

Lardé, Jorge. (1953). "Arqueología Cuzcatleca". En Anales del Museo Nacional "David J. Guzmán”, Tomo IV No.13, pp.75-83. San Salvador, El Salvador, C.A.

Lothrop, Samuel K. (1927). "Pottery types and their sequence in El Salvador". En Indian Notes and Monograpfs (editado por F. W. Hodge), Vol. I No.4, pp.165-220, York, Museum of the American Indian, Heye Foundation, EUA.

Lowe, Gareth W. (1982).“The Izapa sculpture horizon”. En Izapa: An introducción to the Ruins and Monuments (editado por G. W. Lowe, T. A. Lee Jr. y E. Martínez Espinosa) pp. 17 - 42. New World Archaeological Foundation, Brigham Young University, Provo, Utah, E.U.A.

Mendez, Miriam, Hugo Iván Chávez, Julio César Alvarado \& Shione Shibata. (2009). "II. Una formación troncocónica en el municipio de Ayutuxtepeque, San Salvador, El Salvador”. En Informes de hallazgos fortuitos 2009 (Informes entregados al Departamento de Arqueología), pp18-28. Secretaría de Cultura de la Presidencia. El Salvador C.A. 
Morán, Margarita. (2013). Informe final del estudio arqueológico del Proyecto "Nuevo Lourdes Extensión. II Etapa (Etapa I de adquisición). (Informe entregado al Departamento de. Arqueología de la Secretaría de Cultura de la Presidencia). San Salvador, El Salvador, C.A.

Meyer-Abich, Helmut. (1956). "Los Volcanes Activos de Guatemala y El Salvador". En Anales del Servicio Geológico Nacional de El Salvador, Boletín No.3, pp.1-102. San Salvador, El Salvador, C.A.

Ohi, Kuniaki. (2000). "PARTE I Capítulo 1 Desarrollo de las Investigaciones Arqueológicas en el Área. de Casa Blanca". En Chalchuapa (Memoria Final de las Investigaciones. Interdisciplinaria de El Salvador) pp.16-43. Universidad de Estudios Extranjeros. de Kyoto. Kyoto, Japón.

Parsons, Lee Allen. (1986). The origins of Maya art: Monumental Stone sculpture of Kaminaljuyu, Guatemala and the Southern Pacific Coast. Dumbarton Oaks Research Library and Collection, Washington D.C., E.U.A.

Ramos Iglesias, Luisa Massiel. (2018). "Nuevo Lourdes Extensión II Fase II" (Informe Final de la consultoría arqueológica entregado a la Dirección de Arqueología del Ministerio de Cultura. San Salvador, El Salvador C.A.

Sedat., David W., Payson D. Sheets \& Robert J. Sharer. (1978). "Chapter III Excavations in the Casa Blanca Group". En The Prehistory of Chalchuapa Vol.1, pp.88-113. University of Pennsylvania Press. Philadelphia, Pennsylvania, E.U.A.

Sharer Robert J. (1978a). "Chapter II Excavations in the El Trapiche Group". En The Prehistory of Chalchuapa Vol.I Part Three: The Excavations, pp.6187. University of Pennsylvania Press. Philadelphia, Pennsylvania, E.U.A. Sharer Robert J. (1978b) "Chapter II Special Deposits". En The Prehistory of Chalchuapa Vol.I. Part Four: Monuments and Special Deposits, pp.181193. University of Pennsylvania Press. Philadelphia, Pennsylvania, E.U.A.

Sharer Robert J. (1978c). "Chapter II Culture History of Chalchuapa and the Southeastern Maya Highlands". En The Prehistory of Chalchuapa Vol. III Part Two: Conclusions, pp.208-215. University of Pennsylvania Press. Philadelphia, Pennsylvania, E.U.A.

Sheets, Payson D. (1976). "The Terminal Preclassic Lithic Industry of the Southeast Maya Highlands: A Component of the Protoclassic Site-Unit Intrusions in the Lowlands?". En Maya Lithic Studies: Papers From the 1976 Belize Field Symposium, Special Report No.4 (editado por T. R. Hester y N. Hammond) pp.55-70. Center fo Archaeological Research The University of Texas at San Antonio, E.U.A.

Sheets, Payson D. "1. Introduction". En P.D. Sheets (Ed.) Archaeology and Volcanism in Central America, the Zapotitán Valley of El Salvador, pp. 1-13. University of Texas Press. Austin. E.U.A. 
Sheets, Payson D. (2013). Joya de Cerén: Patrimonio Cultural de la Humanidad 193 - 2013. Editorial Universitario de la Universidad de El Salvador. Secretaría de Cultura de la Presidencia. San Salvador, El Salvador, C.A. Shibata, Shione. (2005). " $7^{\mathrm{a}}$ Calle Oriente: Rescate frente al parque arqueológico Tazumal”. En Chalchuapa: Fuentes arqueológicas (Selección de informes realizados entre 1996-2005), pp.121-150. Departamento de Arqueología, Dirección Nacional de Patrimonio Cultural, CONCULTURA. San Salvador, El Salvador, C.A.

Shibata, Shione y Liuba Moran. (2009). Informe del Proyecto El Cambio Temporada 2009 (Informe entregado al Departamento de Arqueología de la Secretaría de Cultura de la Presidencia). San Salvador, El Salvador, C.A. Shibata, Shione y Masakage Murano. (2008). "Investigación arqueológica en el Edificio de las Columnas (B1-1d) de Tazumal, Chalchuapa". En XXI Simposio de Investigaciones Arqueológicas en Guatemala, 2007 (editado por J. P. Laporte, B. Arroyo y H. E. Mejía), Tomo I, pp.1059-1069. Museo Nacional de Arqueología y Etnología. C.D. de Guatemala. Guatemala.

Shibata Shione, Nobuyuki Ito, Hiroshi Minami, Toshio Nakamura \& Etsuko Niu. (2002). "Resultados de las investigaciones arqueológicas en las trincheras 4N y M1 en el área de Casa Blanca Chalchuapa (2000 - 2001)". En XV Simposio de Investigaciones Arqueológicas en Guatemala, 2001 (editado por J. P. Laporte, H. Escobedo y B. Arroyo), Tomo I, pp.1007-1019. Museo Nacional de Arqueología y Etnología. C.D. de Guatemala. Guatemala.

Shibata Shione, Shigeru Kitamura \& Akira Ichikawa. (2010). "Reconsideración del fechamiento de TBJ desde el punto de vista. estratigráfico". En XXIII Simposio de Investigaciones Arqueológicas en Guatemala, 2009 (editado por B. Arroyo, A. Linares y L. Paiz), Tomo II, pp.829-841. Museo Nacional de Arqueología y Etnología. C.D. de Guatemala. Guatemala.

Viera Altamirano, Napoleón. (1951). "Jorge Larde". En ANALES del Museo Nacional "David J. Guzmán", Octubre - Diciembre de 1951 Tomo II No. 8, pp. 10 - 24. Museo Nacional "David J. Guzmán”, El Salvador C.A.

Weber, H.S., G. Wiesemann \& H. Wittekindt (eds.). (1974). Mapa geológica general de la República de El Salvador, 1:500,000. Elaborado por la Misión Geológica Alemana en El Salvador en colaboración con El Centro de Estudios e Investigaciones Geotécnicas (1967-1971). Hanover. Bundesanstalt für Bodenforschung.

Weyl, Richard. (1955). "Las cenizas de pómez en los alrededores de San Salvador". En Comunicaciones Vol.4 (3-4) pp.81-94. Instituto Tropical de Investigaciones Científicas (Universidad de El Salvador). San Salvador, El Salvador C.A.

Williams Howel \& Helmut Meyer-Abich. (1953). "El origen del Lago de Ilopango". En Comunicaciones Vol.2 (1) pp.1-8. Instituto Tropical de Investigaciones Científicas (Universidad de El Salvador). San Salvador, El Salvador C.A. 


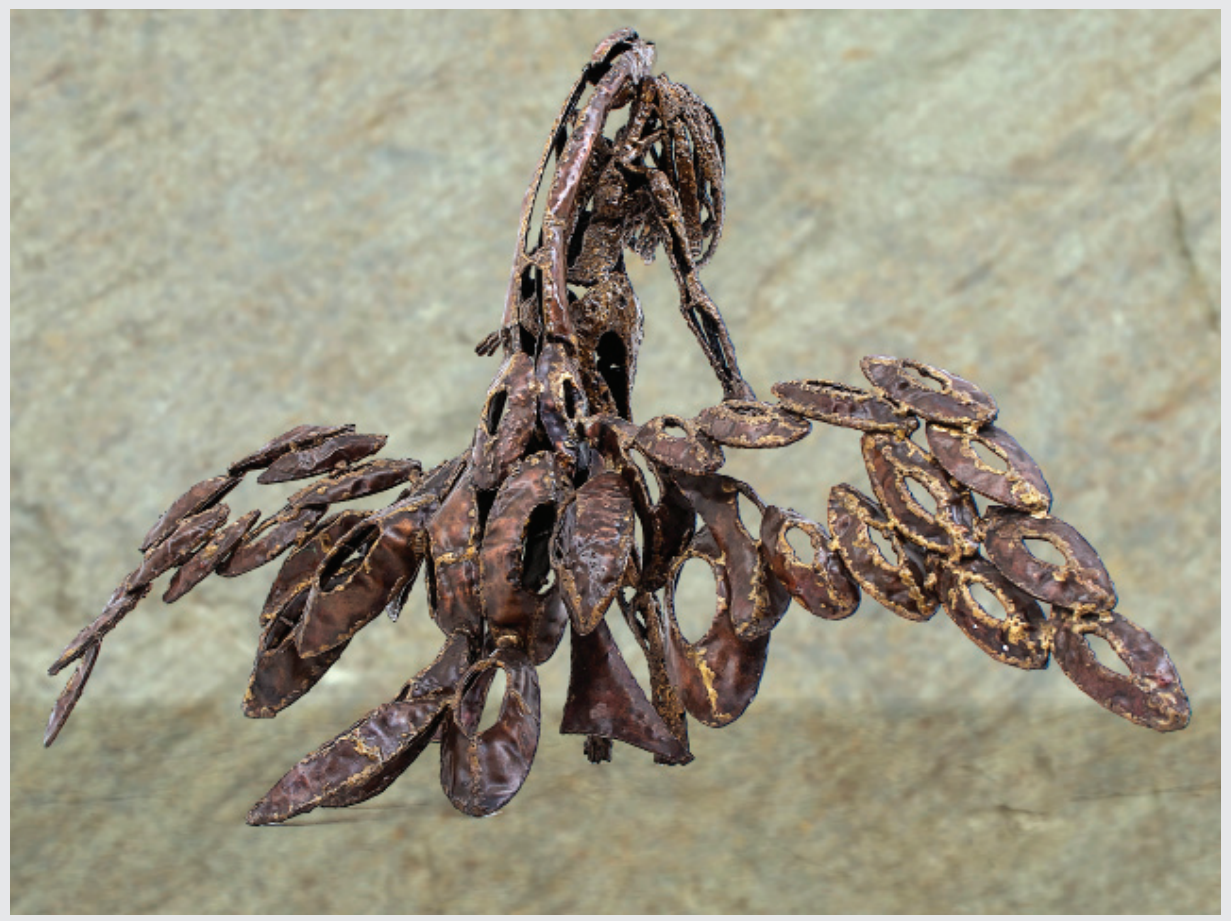

Rubén Martínez Bulnes 